\title{
Highly Enantioselective Hetero-Diels-Alder Reaction of Brassard Diene with Aromatic Aldehydes
}

\author{
Qian Fan, Lili Lin, Jie Liu, Yaozong Huang, Xiaoming Feng*, and Guolin Zhang \\ Key Laboratory of Green Chemistry \& Technology (Sichuan University) Ministry of Education \\ College of Chemistry, Sichuan University, Chengdu 610064 (P. R. China) \\ Fax: (+86)28-85418249 \\ E-mail:xmfeng@scu.edu.cn
}

\section{Supporting information}

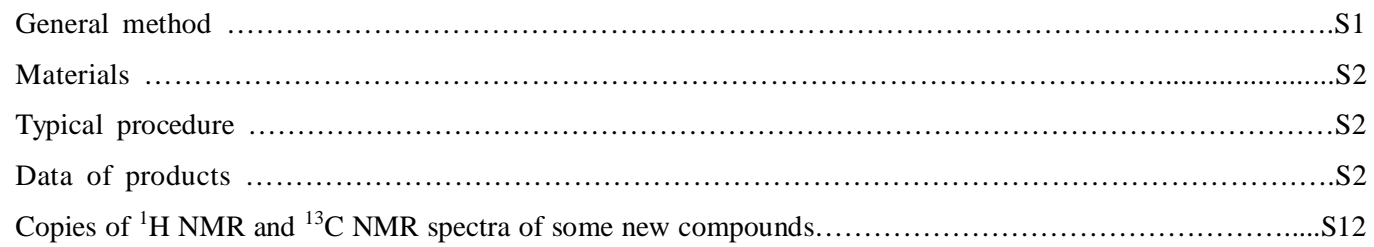

General Method. Unless otherwise noted, all non-aqueous reactions were carried out under an atmosphere of dry nitrogen in dried glassware. All manipulations involving $\mathrm{Ti}(\mathrm{O} i \mathrm{Pr})_{4}$ were processed using standard Schlenck techniques. The ${ }^{1} \mathrm{H}$ and ${ }^{13} \mathrm{C}$ NMR spectra were recorded at $600 \mathrm{MHz}$ and $150 \mathrm{MHz}$ (Bruker Avance), respectively. The chemical shifts are reported in ppm downfield to $\mathrm{CHCl}_{3}(\delta=7.27)$ for ${ }^{1} \mathrm{H}$ NMR and relative to the central $\mathrm{CDCl}_{3}$ resonance $(\delta=77.0)$ for ${ }^{13} \mathrm{C}$ NMR. Coupling constants in ${ }^{1} \mathrm{H}$ NMR are in $\mathrm{Hz}$. HRMS was recorded on BRUKER-APEX-2 (SIMS). Optical rotation data were recorded on Perkin-Elmer Polarimeter-341. The enantiomeric excess (ee) of the products were determined by HPLC using Chiralpak AD-H or Chiralcel OJ columns with hexane/isopropanol as eluent, and the retention times were compared to corresponding racemics samples. 
Materials. Toluene, THF and $\mathrm{Et}_{2} \mathrm{O}$ were distilled freshly from sodium/benzophenone ketyl. $\mathrm{CH}_{2} \mathrm{Cl}_{2}$ was distilled freshly from $\mathrm{CaH}_{2}$. Brassard diene $\mathbf{1 b}$ was prepared from ethyl 3-ethoxybut-2-enoate ${ }^{1}$ according to a literature procedure ${ }^{2}$. Ligands $2 \mathbf{a}-\mathbf{f}$ were prepared $^{3}$ from aminoethanol and substituted salicylaldehydes, purchased from Aldrich or prepared from corresponding phenols ${ }^{3 \mathrm{c}}$. $\mathrm{Ti}(\mathrm{O} i \mathrm{Pr})_{4}$ was distilled and stored as $1.0 \mathrm{M}$ solution in Toluene. All aldehydes were purchased from Acros, Aldrich or Fluka. Liquid aldehydes were distilled in vacuo prior to use, and solid aldehydes were used directly without further purification. Racemics samples of 3a-l were prepared with anhydrous $\mathrm{ZnCl}_{2}$ or $\mathrm{Et}_{2} \mathrm{AlCl}$ as the catalyst ${ }^{4}$.

Typical procedure for HDA reaction of Brassard diene with aromatic aldehydes. $\mathrm{Ti}(\mathrm{O} i \mathrm{Pr})_{4}$ (12.5 $\mu \mathrm{L}, 1 \mathrm{M}$ in toluene, $0.0125 \mathrm{mmol})$ was stirred with $\mathbf{2 a}(5.9 \mathrm{mg}, 0.01375 \mathrm{mmol})$ in $\mathrm{CH}_{2} \mathrm{Cl}_{2}(0.5$ $\mathrm{mL}$ ) at $35^{\circ} \mathrm{C}$ for $1 \mathrm{~h}$ under $\mathrm{N}_{2}$ atmosphere. Then the mixture was cooled to room temperature, and followed by addition of benzaldehyde $(25 \mu \mathrm{L}, 0.25 \mathrm{mmol})$ and $0.5 \mathrm{~mL}$ dry $\mathrm{CH}_{2} \mathrm{Cl}_{2}$. The reaction solution was stirred for $0.5 \mathrm{~h}$, then cooled to $-20^{\circ} \mathrm{C}$, and followed by addition of Brassard diene $\mathbf{1 b}$ $(85 \mu \mathrm{L}, 0.375 \mathrm{mmol})$. The reaction mixture was stirred for $72 \mathrm{~h}$ at $0{ }^{\circ} \mathrm{C}$ before quenched with 5 drops of TFA. After stirring for additional $2 \mathrm{~h}$, the mixture was neutralized with saturated $\mathrm{Na}_{2} \mathrm{CO}_{3}$ $(2 \mathrm{~mL})$, and partitioned between $\mathrm{Et}_{2} \mathrm{O}$ and water twice. The combined organic layers were washed with saturated brine and then dried over anhydrous $\mathrm{Na}_{2} \mathrm{SO}_{4}$ and concentrated in vacuo. The residue was purified by flash chromatography on silica gel (eluent 3:1 petroleum ether: ethyl acetate) afforded the cycloadduct 3a in 71\% yield with $93 \%$ ee (determined by HPLC on Chiralpak AD-H Column, Hexane / isopropanol $95: 5$, flow rate $\left.1.0 \mathrm{~mL} / \mathrm{min}, \mathrm{t}_{\text {major }}=19.83 \mathrm{~min}, \mathrm{t}_{\text {minor }}=23.11 \mathrm{~min}\right) .[\alpha]$ ${ }^{20}=+160^{\circ}\left(\mathrm{c}=0.16\right.$ in $\left.\mathrm{CH}_{2} \mathrm{Cl}_{2}\right)$.

\section{Data of products.}

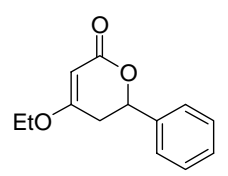

4-ethoxy-5,6-dihydro-6-phenylpyran-2-one (3a)

The ee was determined by HPLC using a Chiralpak AD-H column (95/5 hexane/isopropanol; flow rate $\left.1.0 \mathrm{~mL} / \mathrm{min} ; \mathrm{t}_{\text {major }}=19.8 \mathrm{~min}, \mathrm{t}_{\text {minor }}=23.1 \mathrm{~min}\right)$. $[\alpha]_{\mathrm{D}}{ }^{20}=+160.0^{\circ}\left(\mathrm{c}=0.160, \mathrm{CH}_{2} \mathrm{Cl}_{2}, 93 \%\right.$ ee; $71 \%$ yield $) .{ }^{1} \mathrm{H} \mathrm{NMR} \delta(600 \mathrm{MHz}$, $\mathrm{CDCl}_{3}$ ): 7.43-7.35 (m, 5H; Ph-H), 5.44 (dd, J=12.2, 3.8, 1H; PhCH-O), 5.23 $(\mathrm{d}, J=1.4,1 \mathrm{H} ;=\mathrm{CH}), 4.01\left(\mathrm{~m}, 2 \mathrm{H} ; \mathrm{OCH}_{2}\right), 2.83\left(\mathrm{ddd}, J=17.2,12.2,1.4,1 \mathrm{H} ; \mathrm{CH}_{\mathrm{A}} \mathrm{H}_{\mathrm{B}}\right), 2.60(\mathrm{dd}$, $\left.J=17.2,4.0,1 \mathrm{H} ; \mathrm{CH}_{\mathrm{A}} \mathrm{H}_{\mathrm{B}}\right), 1.41\left(\mathrm{t}, J=7.1,3 \mathrm{H} ; \mathrm{CH}_{3}\right) .{ }^{13} \mathrm{C} \mathrm{NMR} \delta\left(150 \mathrm{MHz}, \mathrm{CDCl}_{3}\right): 172.0,167.3$, 138.5, 128.9, 128.8, 126.2, 91.0, 77.4, 65.2, 35.5, 14.2. HRMS: $(\mathrm{M}+\mathrm{H})^{+}$, calcd 219.1016, found 219.1018 .

1. Smissman, E. E.; Voldeng, N.; J. Org. Chem. 1964, 29, 3161.

2. Savard, J.; Brassard, P.; Tetrahedron Lett. 1979, 20, 4911.

3. (a) Jiang, Y.-Z.; Zhou, X.-G.; Hu, W.-H.; Wu, L.-J.; Mi, A.-Q.; Tetrahedron: Asymmetry 1995, 6, 405; (b) Hayashi, M.; Kaneda, H.; Oguni, N.; Tetrahedron: Asymmetry, 1995, 6, 2511; (c) Gademann, K.; Chavez, D. E.; Jacobsen, E. N.; Angew. Chem. Int. Ed. Engl. 2002, 41, 3059; Angew. Chem. 2002, 114, 3185.

4. Midland, M. M.; Koops, R. W.; J. Org. Chem. 1990, 55, 5058. 

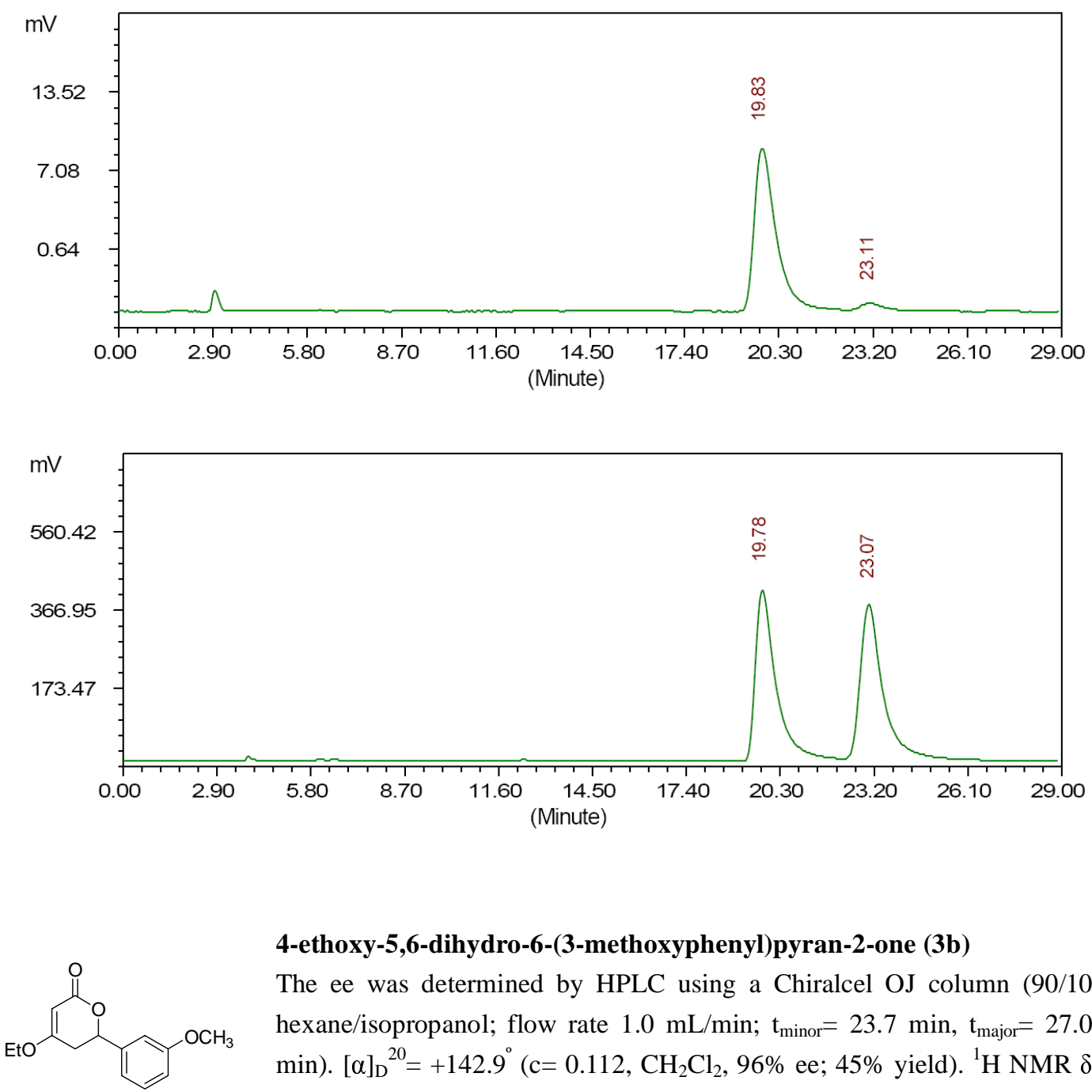

4-ethoxy-5,6-dihydro-6-(3-methoxyphenyl)pyran-2-one (3b)

The ee was determined by HPLC using a Chiralcel OJ column (90/10 hexane/isopropanol; flow rate $1.0 \mathrm{~mL} / \mathrm{min} ; \mathrm{t}_{\text {minor }}=23.7 \mathrm{~min}, \mathrm{t}_{\text {major }}=27.0$ $\min ) .[\alpha]_{\mathrm{D}}{ }^{20}=+142.9^{\circ}$ (c=0.112, $\mathrm{CH}_{2} \mathrm{Cl}_{2}, 96 \%$ ee; $45 \%$ yield). ${ }^{1} \mathrm{H}$ NMR $\delta$ (600MHz, $\left.\mathrm{CDCl}_{3}\right): 7.32-6.89$ (m, 5H; Ph-H), $5.42(\mathrm{dd}, J=12.2,3.8,1 \mathrm{H}$; $\mathrm{PhCH}-\mathrm{O}), 5.22(\mathrm{~s}, 1 \mathrm{H} ;=\mathrm{CH}), 4.00\left(\mathrm{~m}, 2 \mathrm{H} ; \mathrm{OCH}_{2}\right), 3.83\left(\mathrm{~s}, 3 \mathrm{H} ; \mathrm{OCH}_{3}\right), 2.82(\mathrm{dd}, J=17.2,12.2,1 \mathrm{H}$; $\mathrm{CH}_{\mathrm{A}} \mathrm{H}_{\mathrm{B}}$ ), $2.60\left(\mathrm{dd}, J=17.2,3.8,1 \mathrm{H} ; \mathrm{CH}_{\mathrm{A}} \mathrm{H}_{\mathrm{B}}\right), 1.41$ (t, $\left.J=7.1,3 \mathrm{H} ; \mathrm{CH}_{3}\right) .{ }^{13} \mathrm{C}$ NMR $\delta(150 \mathrm{MHz}$, $\left.\mathrm{CDCl}_{3}\right)$ : 172.0, 167.3, 160.1, 140.1, 129.9, 118.3, 114.4, 111.6, 91.0, 65.2, 55.5, 35.5, 14.2. HRMS: $(\mathrm{M}+\mathrm{H})^{+}$, calcd 249.1121, found 249.1125 .

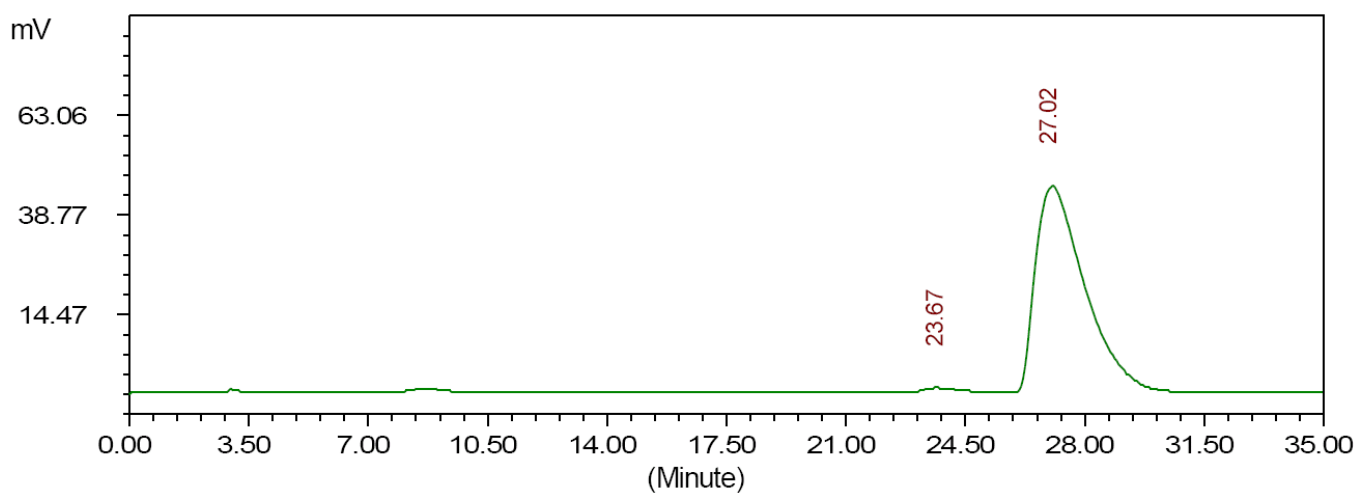



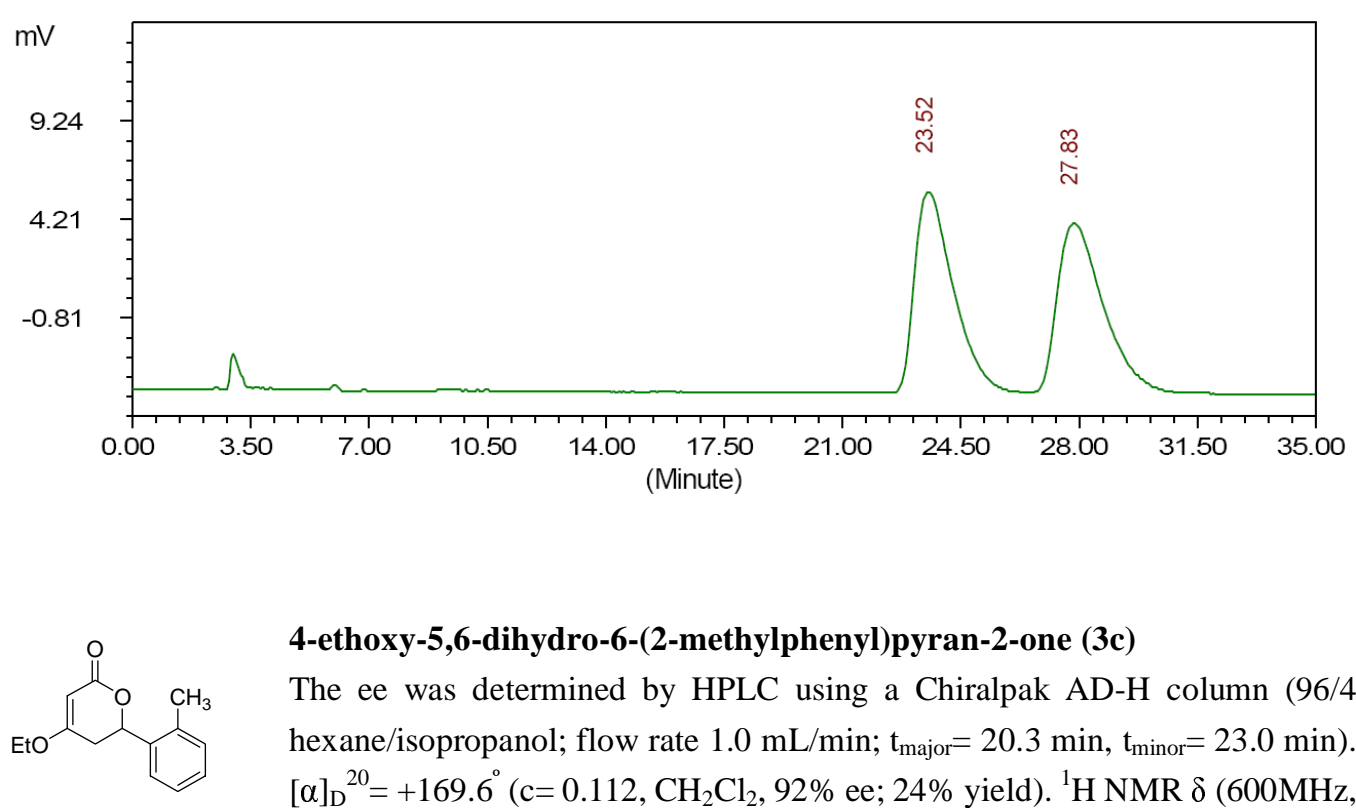

\section{4-ethoxy-5,6-dihydro-6-(2-methylphenyl)pyran-2-one (3c)}

The ee was determined by HPLC using a Chiralpak AD-H column (96/4 hexane/isopropanol; flow rate $1.0 \mathrm{~mL} / \mathrm{min} ; \mathrm{t}_{\text {major }}=20.3 \mathrm{~min}, \mathrm{t}_{\mathrm{minor}}=23.0 \mathrm{~min}$ ). $[\alpha]_{\mathrm{D}}{ }^{20}=+169.6^{\circ}\left(\mathrm{c}=0.112, \mathrm{CH}_{2} \mathrm{Cl}_{2}, 92 \%\right.$ ee; $24 \%$ yield $) .{ }^{1} \mathrm{H}$ NMR $\delta(600 \mathrm{MHz}$, $\mathrm{CDCl}_{3}$ ): 7.53-7.19 (m, 5H; Ph-H), 5.63 (dd, $\left.J=12.6,3.6,1 \mathrm{H} ; \mathrm{PhCH}-\mathrm{O}\right), 5.24$ $(\mathrm{d}, J=1.3,1 \mathrm{H} ;=\mathrm{CH}), 4.02\left(\mathrm{~m}, 2 \mathrm{H} ; \mathrm{OCH}_{2}\right), 2.82\left(\mathrm{ddd}, J=17.2,12.7,1.4,1 \mathrm{H} ; \mathrm{CH}_{\mathrm{A}} \mathrm{H}_{\mathrm{B}}\right), 2.52(\mathrm{dd}, J=$ 17.3, 3.6, $\left.1 \mathrm{H} ; \mathrm{CH}_{\mathrm{A}} \mathrm{H}_{\mathrm{B}}\right), 2.38\left(\mathrm{~s}, 3 \mathrm{H} ; \mathrm{PhCH}_{3}\right), 1.42\left(\mathrm{t}, J=7.1,3 \mathrm{H} ; \mathrm{CH}_{3}\right) .{ }^{13} \mathrm{C}$ NMR $\delta(150 \mathrm{MHz}$, $\mathrm{CDCl}_{3}$ ): 172.2, 167.5, 136.5, 135.0, 130.9, 128.7, 126.7, 126.3, 90.9, 74.8, 65.2, 34.4, 19.3, 14.3. HRMS: $(\mathrm{M}+\mathrm{H})^{+}$, calcd 233.1172, found 233.1175.
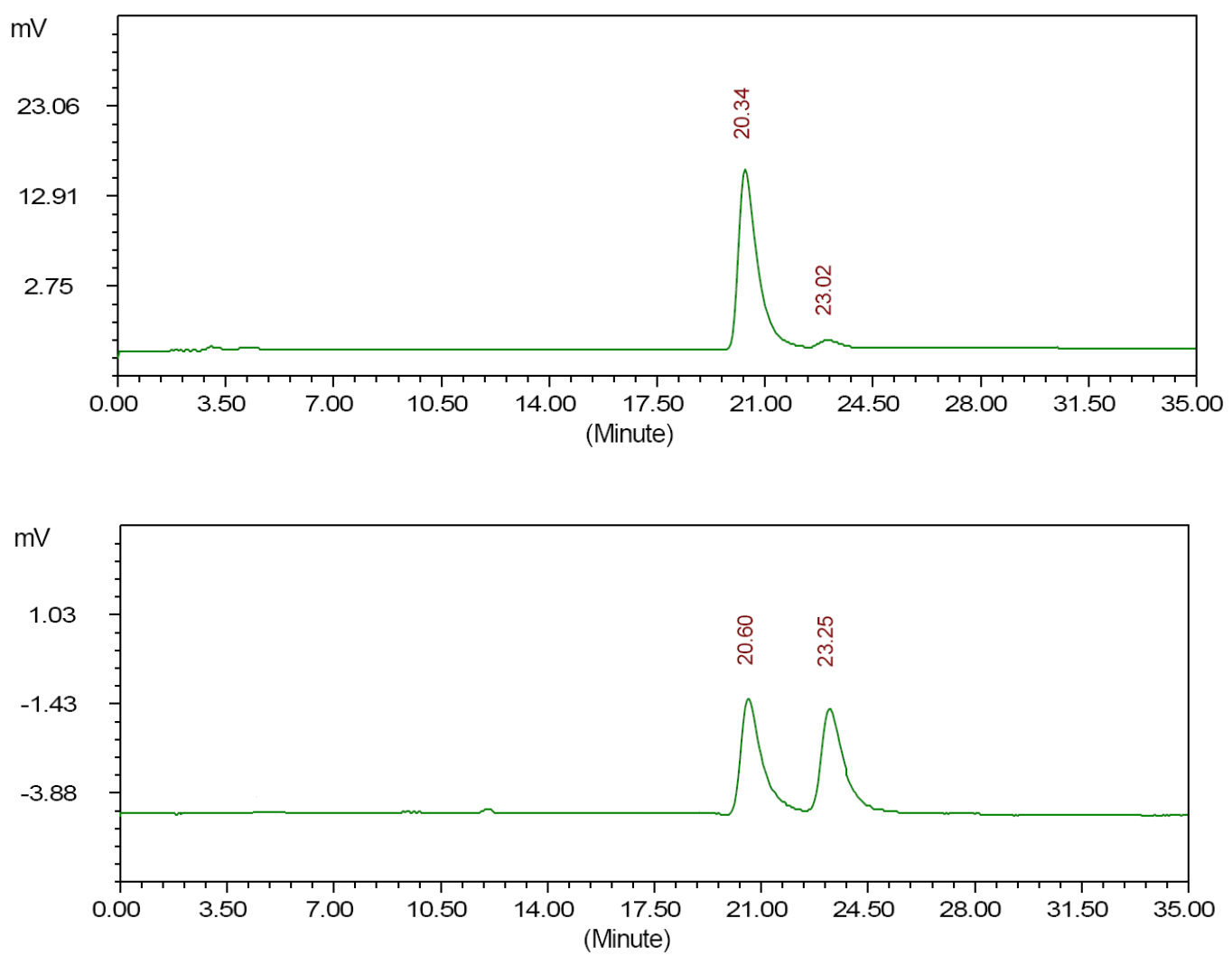


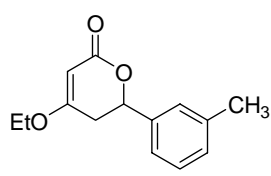

4-ethoxy-5,6-dihydro-6-(3-methylphenyl)pyran-2-one (3d)

The ee was determined by HPLC using a Chiralpak AD-H column $(95 / 5$ hexane/isopropanol; flow rate $1.0 \mathrm{~mL} / \mathrm{min} ; \mathrm{t}_{\text {major }}=15.6 \mathrm{~min}, \mathrm{t}_{\text {minor }}=18.0$ $\min )$. $[\alpha]_{\mathrm{D}}{ }^{20}=+173.2^{\circ}\left(\mathrm{c}=0.112, \mathrm{CH}_{2} \mathrm{Cl}_{2}, 93 \%\right.$ ee; $53 \%$ yield $) .{ }^{1} \mathrm{H} \mathrm{NMR} \delta$ $\left(600 \mathrm{MHz}, \mathrm{CDCl}_{3}\right)$ : 7.30-7.26 (m, 2H; Ph-H), 7.20-7.17 (m, 2H; Ph-H), $5.40(\mathrm{dd}, J=12.2,3.8,1 \mathrm{H} ; \mathrm{PhCH}-\mathrm{O}), 5.22(\mathrm{~d}, J=1.2,1 \mathrm{H} ;=\mathrm{CH}), 4.00\left(\mathrm{~m}, 2 \mathrm{H} ; \mathrm{OCH}_{2}\right), 2.82(\mathrm{ddd}$, $\left.J=17.2,12.3,1.3,1 \mathrm{H} ; \mathrm{CH}_{\mathrm{A}} \mathrm{H}_{\mathrm{B}}\right), 2.59\left(\mathrm{dd}, J=17.2,3.8,1 \mathrm{H} ; \mathrm{CH}_{\mathrm{A}} \mathrm{H}_{\mathrm{B}}\right), 2.38\left(\mathrm{~s}, 3 \mathrm{H} ; \mathrm{PhCH}_{3}\right), 1.41(\mathrm{t}$, $\left.J=7.1,3 \mathrm{H} ; \mathrm{CH}_{3}\right) .{ }^{13} \mathrm{C}$ NMR $\delta\left(150 \mathrm{MHz}, \mathrm{CDCl}_{3}\right): 172.0,167.4,138.7,138.5,129.5,128.7,126.9$, 123.2, 91.0, 77.4, 65.1, 35.5, 21.6, 14.2. HRMS: $(\mathrm{M}+\mathrm{H})^{+}$, calcd 233.1172, found 233.1171.
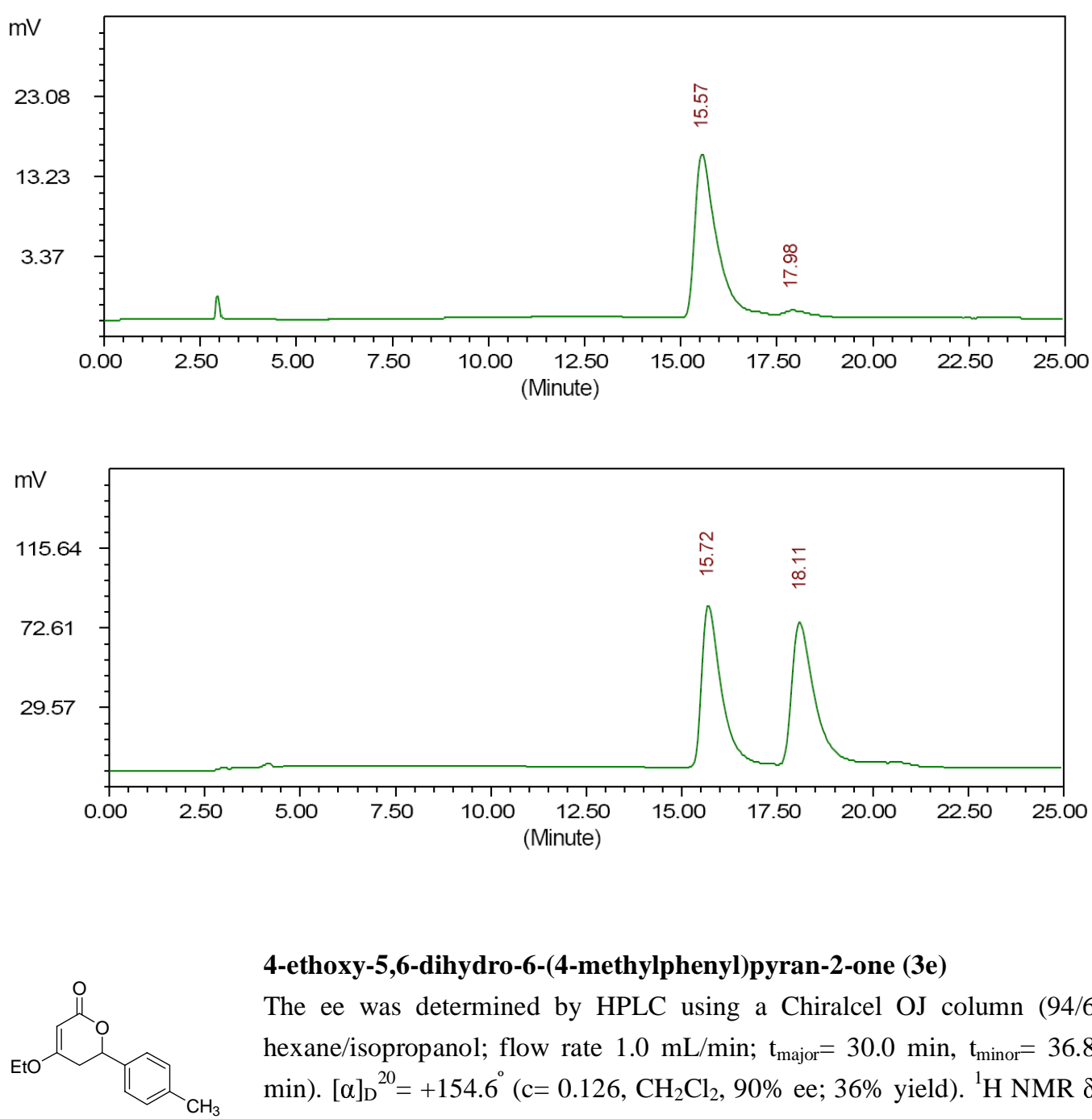

\section{4-ethoxy-5,6-dihydro-6-(4-methylphenyl)pyran-2-one (3e)}

The ee was determined by HPLC using a Chiralcel OJ column $(94 / 6$ hexane/isopropanol; flow rate $1.0 \mathrm{~mL} / \mathrm{min} ; \mathrm{t}_{\text {major }}=30.0 \mathrm{~min}, \mathrm{t}_{\text {minor }}=36.8$ $\min ) .[\alpha]_{\mathrm{D}}{ }^{20}=+154.6^{\circ}\left(\mathrm{c}=0.126, \mathrm{CH}_{2} \mathrm{Cl}_{2}, 90 \%\right.$ ee; $36 \%$ yield $) .{ }^{1} \mathrm{H}$ NMR $\delta$ $\left(600 \mathrm{MHz}, \mathrm{CDCl}_{3}\right): 7.31-7.20(\mathrm{~m}, 4 \mathrm{H} ; \mathrm{Ph}-\mathrm{H}), 5.40(\mathrm{dd}, J=12.2,3.8,1 \mathrm{H}$;

PhCH-O), $5.22(\mathrm{~s}, 1 \mathrm{H} ;=\mathrm{CH}), 4.00\left(\mathrm{~m}, 2 \mathrm{H} ; \mathrm{OCH}_{2}\right), 2.82\left(\mathrm{dd}, J=17.2,12.3,1 \mathrm{H} ; \mathrm{CH}_{\mathrm{A}} \mathrm{H}_{\mathrm{B}}\right), 2.57(\mathrm{dd}$, $\left.J=17.2,3.8,1 \mathrm{H} ; \mathrm{CH}_{\mathrm{A}} \mathrm{H}_{\mathrm{B}}\right), 2.37\left(\mathrm{~s}, 3 \mathrm{H} ; \mathrm{PhCH}_{3}\right), 1.42\left(\mathrm{t}, J=7.1,3 \mathrm{H} ; \mathrm{CH}_{3}\right) .{ }^{13} \mathrm{C} \mathrm{NMR} \delta(150 \mathrm{MHz}$, $\left.\mathrm{CDCl}_{3}\right): 172.0,167.5,138.6,135.6,129.5,126.2,91.0,77.4,65.1,35.5,21.4,14.2$. HRMS: $(\mathrm{M}+\mathrm{H})^{+}$, calcd 233.1172, found 233.1176. 

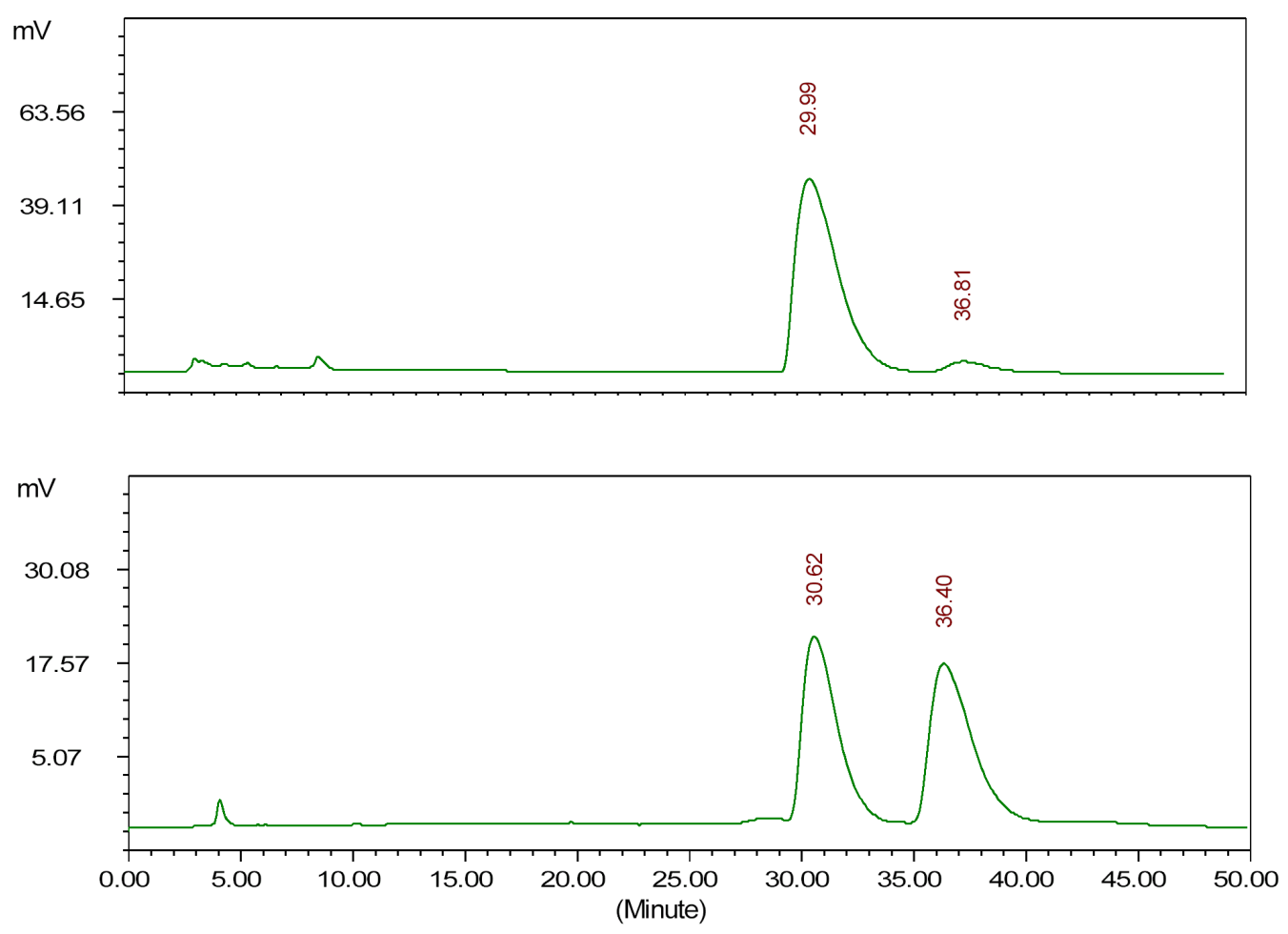

\section{4-ethoxy-5,6-dihydro-6-(2-chlorophenyl)pyran-2-one (3f)}

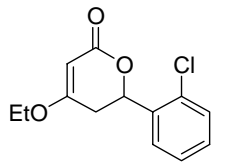

The ee was determined by HPLC using a Chiralpak AD-H column (98/2 hexane/isopropanol; flow rate $\left.1.0 \mathrm{~mL} / \mathrm{min} ; \mathrm{t}_{\text {major }}=26.1 \mathrm{~min}, \mathrm{t}_{\text {minor }}=29.9 \mathrm{~min}\right)$. $[\alpha]_{\mathrm{D}}{ }^{20}=+270.2^{\circ}\left(\mathrm{c}=0.124, \mathrm{CH}_{2} \mathrm{Cl}_{2}, 99 \%\right.$ ee; $70 \%$ yield $) .{ }^{1} \mathrm{H} \mathrm{NMR} \delta(600 \mathrm{MHz}$, $\left.\mathrm{CDCl}_{3}\right)$ : 7.69-7.67 (m, 1H; Ph-H), 7.39-7.35 (m, 2H; Ph-H), 7.32-7.29 (m, 1H;

Ph-H), 5.80 (dd, $J=12.3,3.8,1 \mathrm{H} ; \mathrm{PhCH}-\mathrm{O}), 5.24(\mathrm{~d}, J=1.3,1 \mathrm{H} ;=\mathrm{CH}), 4.02\left(\mathrm{~m}, 2 \mathrm{H} ; \mathrm{OCH}_{2}\right), 2.78$ (ddd, $J=17.2,12.2,3.8,2 \mathrm{H} ; \mathrm{CH}_{\mathrm{A}} \mathrm{H}_{\mathrm{B}}$ ), 2.62 (ddd, $J=17.2,12.2,1.4,1 \mathrm{H} ; \mathrm{CH}_{\mathrm{A}} \mathrm{H}_{\mathrm{B}}$ ), 1.42 (t, $J=7.1$, $\left.3 \mathrm{H} ; \mathrm{CH}_{3}\right) .{ }^{13} \mathrm{C} \mathrm{NMR} \delta\left(150 \mathrm{MHz}, \mathrm{CDCl}_{3}\right): 172.0,167.2,136.5,131.5,129.8,127.7,127.6,90.8$, 74.4, 65.3, 34.1, 14.2. HRMS: $(\mathrm{M}+\mathrm{H})^{+}$, calcd 253.0626, found 253.0632 .

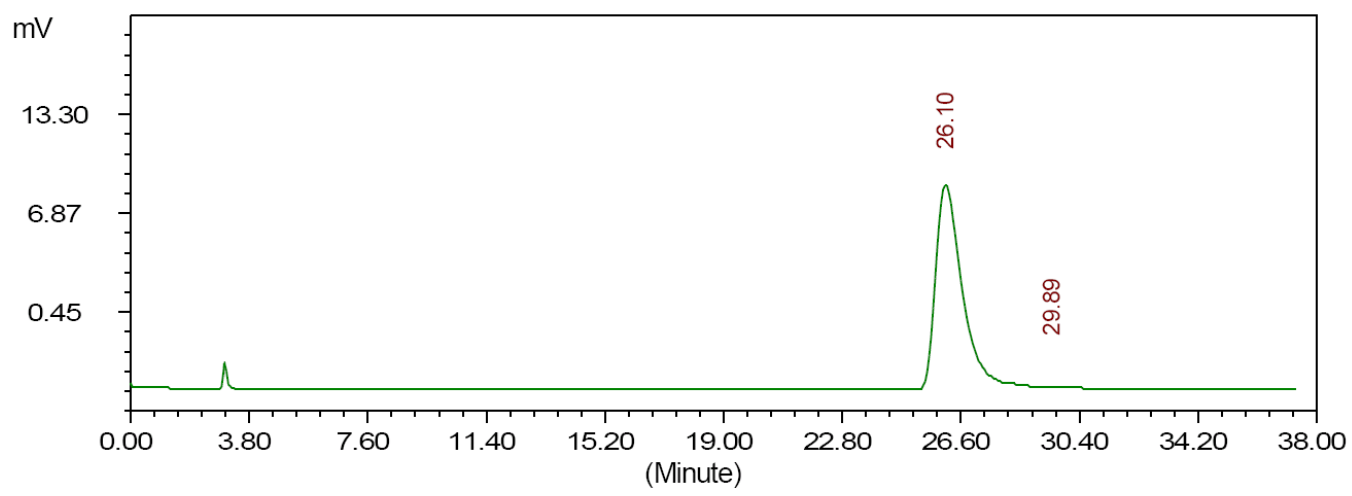



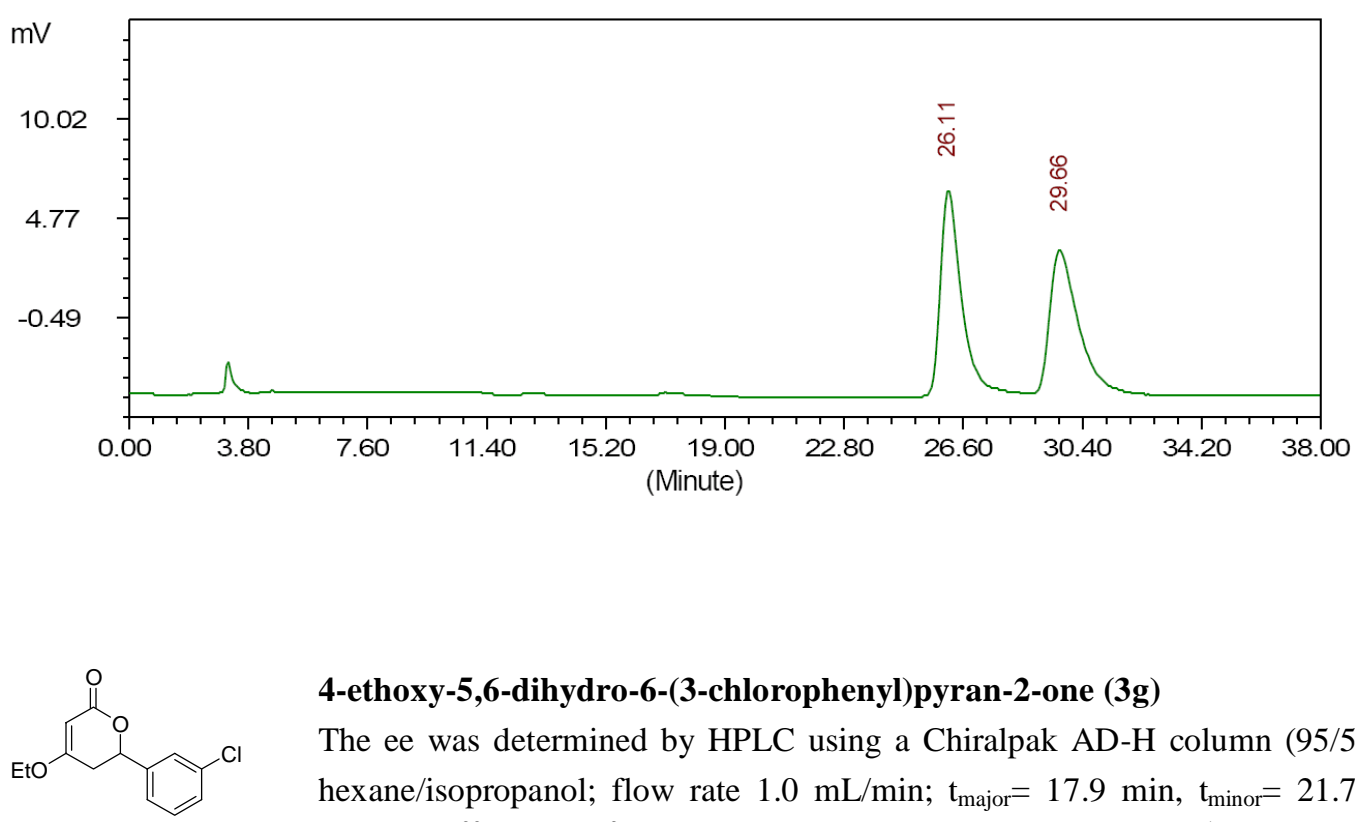

4-ethoxy-5,6-dihydro-6-(3-chlorophenyl)pyran-2-one (3g)

The ee was determined by HPLC using a Chiralpak AD-H column $(95 / 5$

hexane/isopropanol; flow rate $1.0 \mathrm{~mL} / \mathrm{min} ; \mathrm{t}_{\text {major }}=17.9 \mathrm{~min}, \mathrm{t}_{\text {minor }}=21.7$ $\min$ ). $[\alpha]_{\mathrm{D}}{ }^{20}=+161.0^{\circ}$ (c=0.118, $\mathrm{CH}_{2} \mathrm{Cl}_{2}, 90 \%$ ee; $70 \%$ yield). ${ }^{1} \mathrm{H}$ NMR $\delta$ $\left(600 \mathrm{MHz}, \mathrm{CDCl}_{3}\right): 7.44(\mathrm{~s}, 1 \mathrm{H} ; \mathrm{Ph}-\mathrm{H}), 7.34-7.29(\mathrm{~m}, 3 \mathrm{H} ; \mathrm{Ph}-\mathrm{H}), 5.41$ (dd, J=12.2, 3.9, 1H; PhCH-O), $5.22(\mathrm{~d}, J=1.5,1 \mathrm{H} ;=\mathrm{CH}), 4.01\left(\mathrm{~m}, 2 \mathrm{H} ; \mathrm{OCH}_{2}\right), 2.79$ (ddd, $J=17.2,12.2,1.4,1 \mathrm{H}$; $\left.\mathrm{CH}_{\mathrm{A}} \mathrm{H}_{\mathrm{B}}\right), 2.61\left(\mathrm{dd}, J=17.1,4.0,1 \mathrm{H} ; \mathrm{CH}_{\mathrm{A}} \mathrm{H}_{\mathrm{B}}\right), 1.41\left(\mathrm{t}, J=7.1,1 \mathrm{H} ; \mathrm{CH}_{3}\right) .{ }^{13} \mathrm{C}$ NMR $\delta(150 \mathrm{MHz}$, $\left.\mathrm{CDCl}_{3}\right): 171.7,166.8,140.6,134.9,130.2,128.9,126.4,124.2,91.0,76.4,65.3,35.4,14.2$. HRMS: $(\mathrm{M}+\mathrm{H})^{+}$, calcd 253.0626, found 253.0624 .
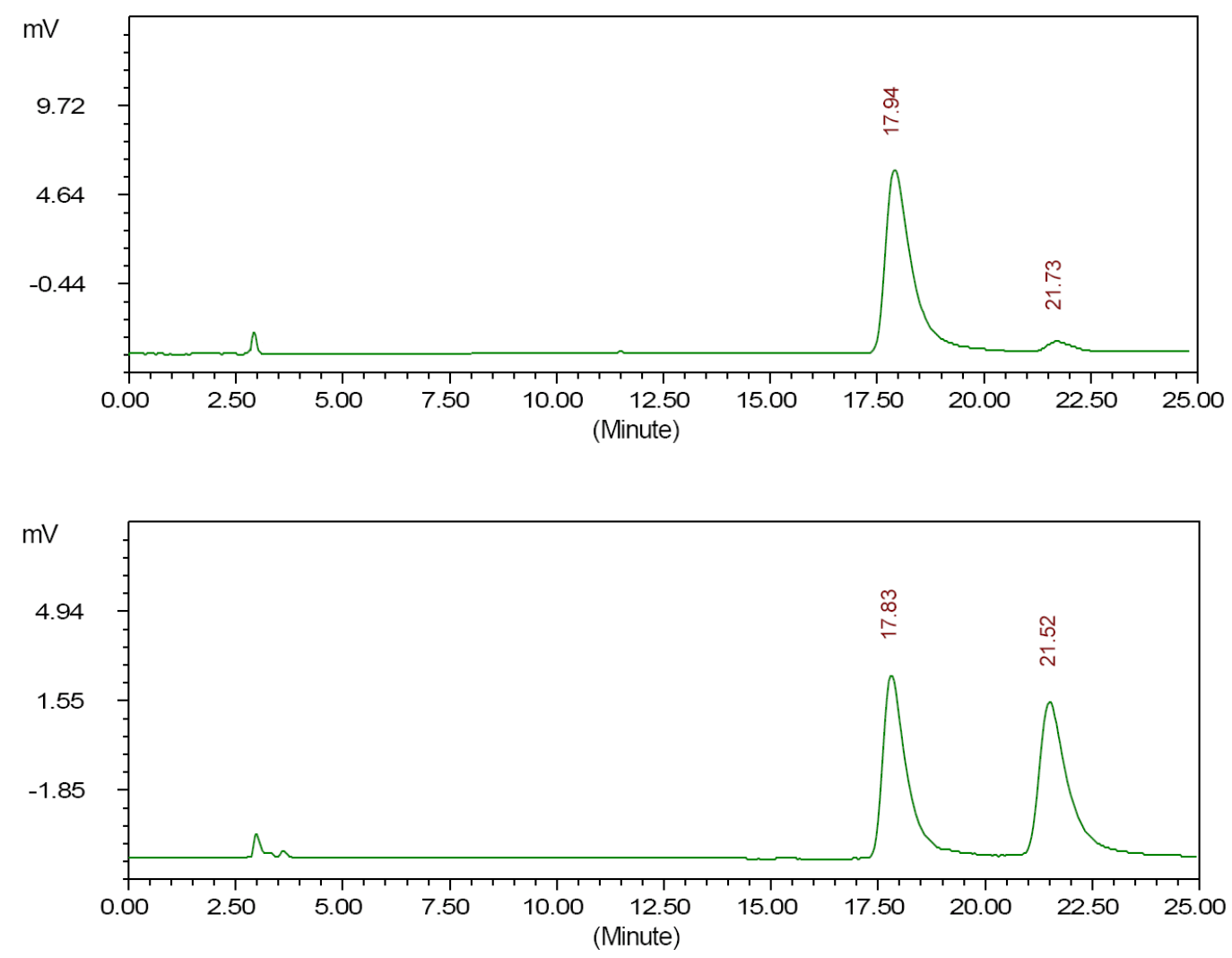


\section{4-ethoxy-5,6-dihydro-6-(4-chlorophenyl)pyran-2-one (3h)}

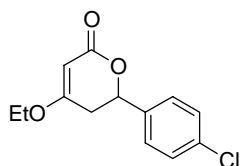

The ee was determined by HPLC using a Chiralpak AD-H column (95/5 hexane/isopropanol; flow rate $1.0 \mathrm{~mL} / \mathrm{min} ; \mathrm{t}_{\text {major }}=23.9 \mathrm{~min}, \mathrm{t}_{\mathrm{minor}}=29.1 \mathrm{~min}$ ). $[\alpha]_{\mathrm{D}}{ }^{20}=+151.9^{\circ}\left(\mathrm{c}=0.104, \mathrm{CH}_{2} \mathrm{Cl}_{2}, 97 \%\right.$ ee; $87 \%$ yield $) .{ }^{1} \mathrm{H} \mathrm{NMR} \delta(600 \mathrm{MHz}$, $\mathrm{CDCl}_{3}$ ): 7.39-7.35 (m, 4H; Ph-H), 5.41 (dd, $\left.J=12.1,3.8,1 \mathrm{H} ; \mathrm{PhCH}-\mathrm{O}\right), 5.22$

$(\mathrm{s}, 1 \mathrm{H} ;=\mathrm{CH}), 4.00\left(\mathrm{~m}, 2 \mathrm{H} ; \mathrm{OCH}_{2}\right), 2.77\left(\mathrm{dd}, J=17.2,12.1,1 \mathrm{H} ; \mathrm{CH}_{\mathrm{A}} \mathrm{H}_{\mathrm{B}}\right), 2.59(\mathrm{dd}, J=17.2,3.9,1 \mathrm{H}$; $\left.\mathrm{CH}_{\mathrm{A}} \mathrm{H}_{\mathrm{B}}\right), 1.40\left(\mathrm{t}, J=7.0,3 \mathrm{H} ; \mathrm{CH}_{3}\right) .{ }^{13} \mathrm{C} \mathrm{NMR} \delta\left(150 \mathrm{MHz}, \mathrm{CDCl}_{3}\right): 171.7,167.0,137.1,134.6$, 129.1, 127.6, 91.0, 76.6, 65.3, 35.4, 14.2. HRMS: $(\mathrm{M}+\mathrm{H})^{+}$, calcd 253.0626, found 253.0630.
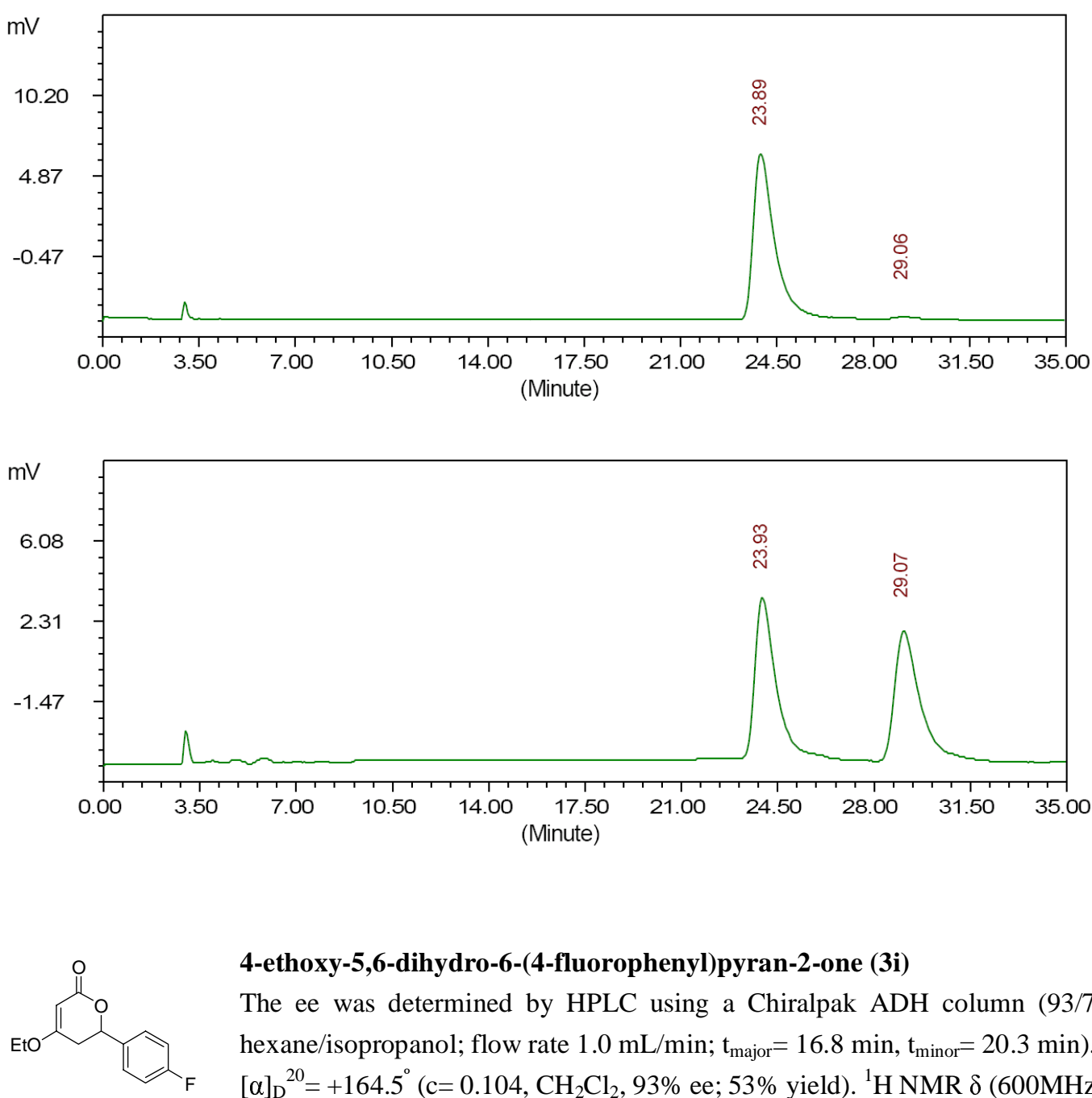

\section{4-ethoxy-5,6-dihydro-6-(4-fluorophenyl)pyran-2-one (3i)}

The ee was determined by HPLC using a Chiralpak ADH column (93/7 hexane/isopropanol; flow rate $\left.1.0 \mathrm{~mL} / \mathrm{min} ; \mathrm{t}_{\text {major }}=16.8 \mathrm{~min}, \mathrm{t}_{\text {minor }}=20.3 \mathrm{~min}\right)$. $[\alpha]_{\mathrm{D}}{ }^{20}=+164.5^{\circ}\left(\mathrm{c}=0.104, \mathrm{CH}_{2} \mathrm{Cl}_{2}, 93 \%\right.$ ee; $53 \%$ yield $) .{ }^{1} \mathrm{H} \mathrm{NMR} \delta(600 \mathrm{MHz}$,

$\mathrm{CDCl}_{3}$ ): 7.41-7.39 (m, 2H; Ph-H), 7.10-7.07 (m, 2H; Ph-H), 5.41 (dd, J= 12.2, 3.8, 1H; PhCH-O), $5.21(\mathrm{~d}, J=1.5,1 \mathrm{H} ;=\mathrm{CH}), 4.00\left(\mathrm{~m}, 2 \mathrm{H} ; \mathrm{OCH}_{2}\right), 2.79\left(\mathrm{ddd}, J=17.1,12.2,1.5,1 \mathrm{H} ; \mathrm{CH}_{\mathrm{A}} \mathrm{H}_{\mathrm{B}}\right), 2.57$ $\left(\mathrm{dd}, J=17.1,3.8,1 \mathrm{H} ; \mathrm{CH}_{\mathrm{A}} \mathrm{H}_{\mathrm{B}}\right), 1.40\left(\mathrm{t}, J=7.0,3 \mathrm{H} ; \mathrm{CH}_{3}\right) .{ }^{13} \mathrm{C} \mathrm{NMR} \delta\left(150 \mathrm{MHz}, \mathrm{CDCl}_{3}\right): 171.8$, $167.1,162.9\left(\mathrm{~d}, J_{C-F}^{l}=245.2,1 \mathrm{C}\right), 134.4\left(\mathrm{~d}, J_{C-F}^{4}=3.4,1 \mathrm{C}\right), 128.1\left(\mathrm{~d}, J_{C-F}^{2}=8.0,1 \mathrm{C}\right), 115.8(\mathrm{~d}$, $\left.J_{C-F}^{3}=21.7,1 \mathrm{C}\right), 91.0,76.7,65.2,35.5,14.2$. HRMS: $(\mathrm{M}+\mathrm{H})^{+}$, calcd 237.0921, found 237.0916. 

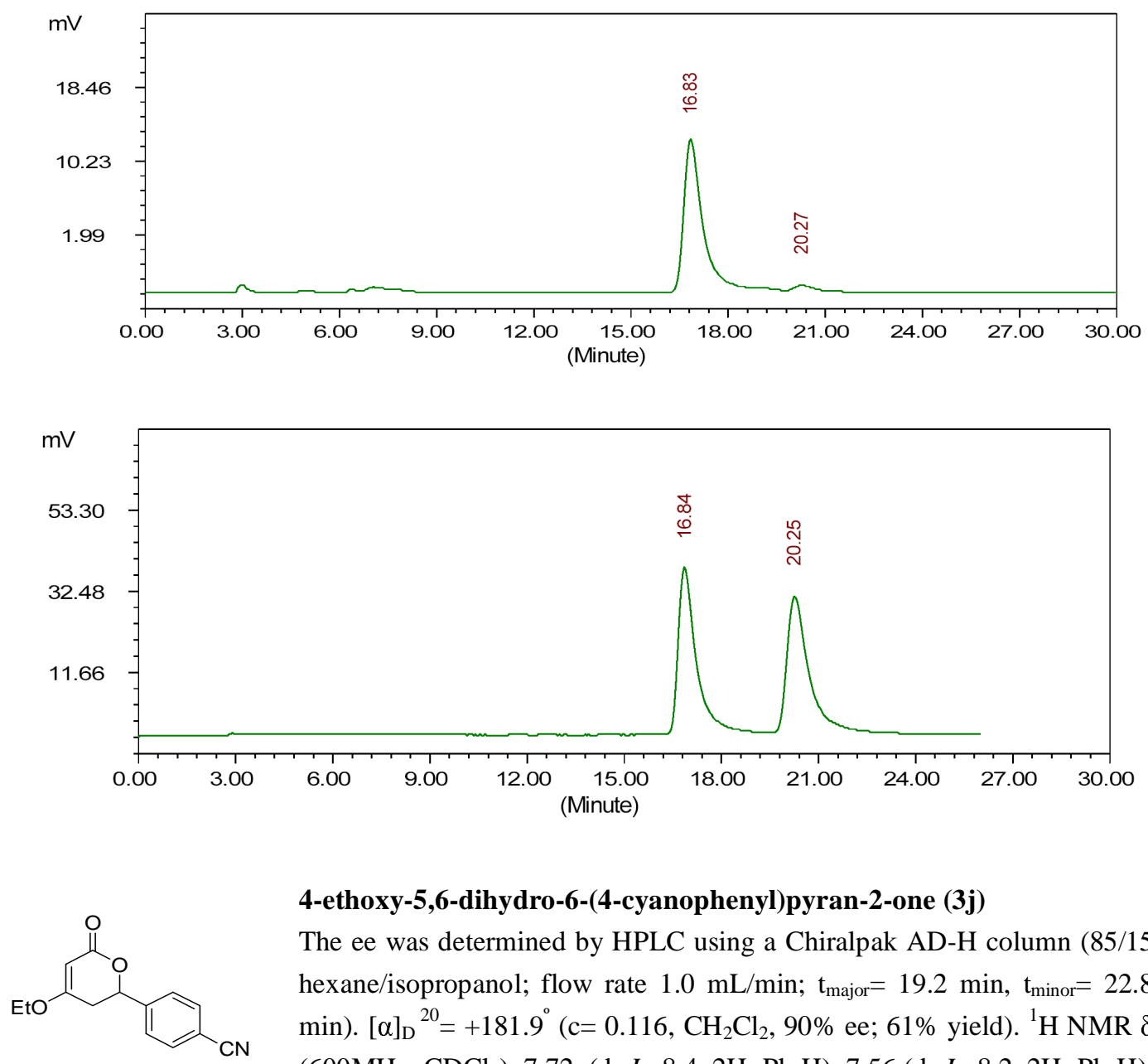

\section{4-ethoxy-5,6-dihydro-6-(4-cyanophenyl)pyran-2-one (3j)}

The ee was determined by HPLC using a Chiralpak AD-H column (85/15 hexane/isopropanol; flow rate $1.0 \mathrm{~mL} / \mathrm{min} ; \mathrm{t}_{\text {major }}=19.2 \mathrm{~min}, \mathrm{t}_{\text {minor }}=22.8$ $\min ) .[\alpha]_{\mathrm{D}}{ }^{20}=+181.9^{\circ}$ (c=0.116, $\mathrm{CH}_{2} \mathrm{Cl}_{2}, 90 \%$ ee; $61 \%$ yield). ${ }^{1} \mathrm{H}$ NMR $\delta$ (600MHz, $\left.\mathrm{CDCl}_{3}\right): 7.72$, (d, $\left.J=8.4,2 \mathrm{H} ; \mathrm{Ph}-\mathrm{H}\right), 7.56$ (d, $\left.J=8.2,2 \mathrm{H} ; \mathrm{Ph}-\mathrm{H}\right)$, 5.49 (dd, $J=12.0,3.9,1 \mathrm{H} ; \mathrm{PhCH}-\mathrm{O}), 5.24(\mathrm{~d}, J=1.3,1 \mathrm{H} ;=\mathrm{CH}), 4.02(\mathrm{~m}$, $2 \mathrm{H} ; \mathrm{OCH}_{2}$ ), 2.76 (ddd, $\left.J=17.1,12.0,1.3,1 \mathrm{H} ; \mathrm{CH}_{\mathrm{A}} \mathrm{H}_{\mathrm{B}}\right), 2.64$ (dd, $\left.J=17.1,3.9,1 \mathrm{H} ; \mathrm{CH}_{\mathrm{A}} \mathrm{H}_{\mathrm{B}}\right), 1.42$ (t, $\left.J=7.1,3 \mathrm{H} ; \mathrm{CH}_{3}\right) .{ }^{13} \mathrm{C}$ NMR $\delta\left(150 \mathrm{MHz}, \mathrm{CDCl}_{3}\right): 171.4,166.4,143.7,132.8,126.8,118.5,112.7$, 91.0, 76.2, 65.4, 35.3, 14.2. HRMS: $(\mathrm{M}+\mathrm{H})^{+}$, calcd 244.0968, found 244.0976.

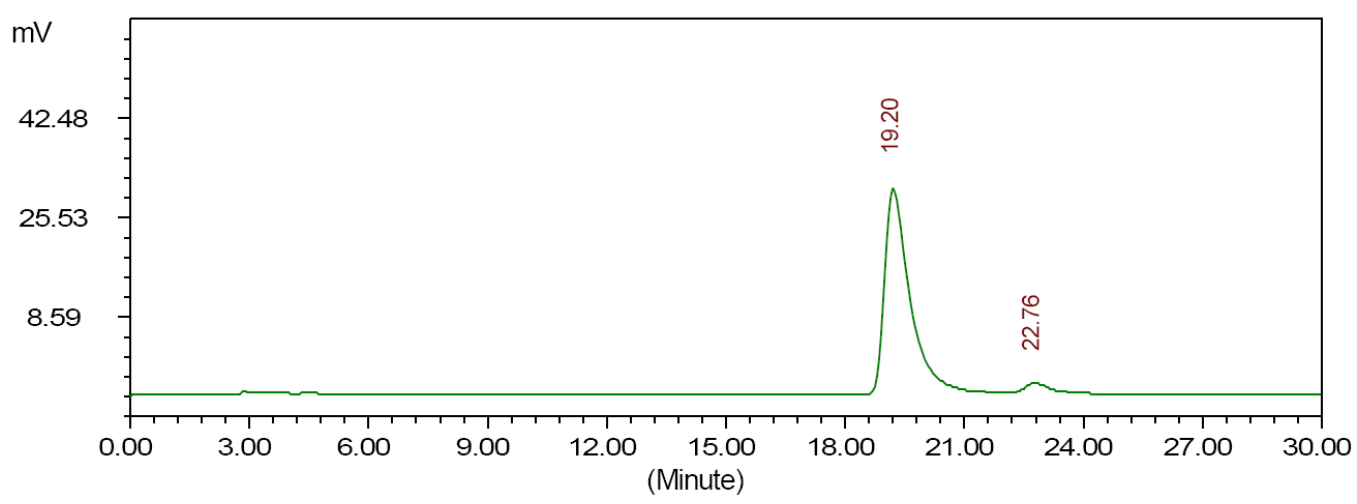



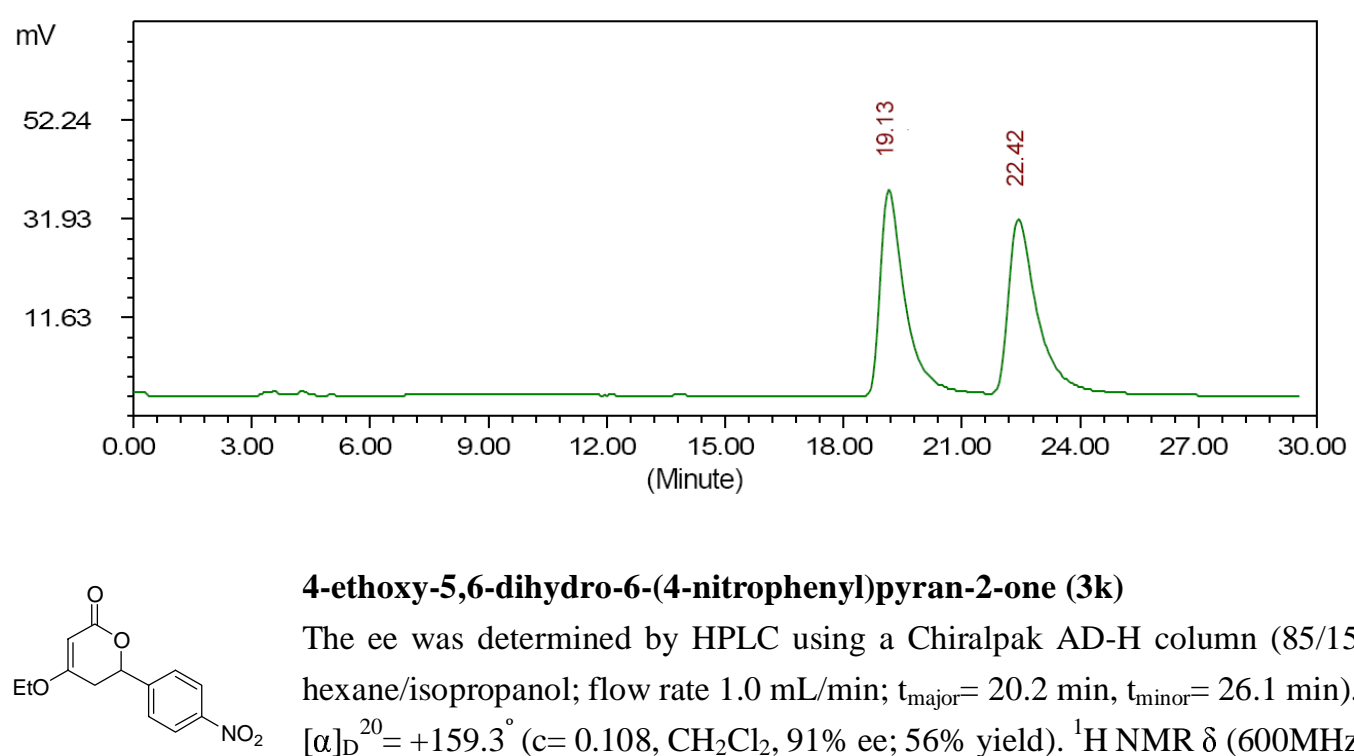

4-ethoxy-5,6-dihydro-6-(4-nitrophenyl)pyran-2-one (3k)

The ee was determined by HPLC using a Chiralpak AD-H column (85/15 hexane/isopropanol; flow rate $1.0 \mathrm{~mL} / \mathrm{min}$; $\mathrm{t}_{\text {major }}=20.2 \mathrm{~min}$, $\mathrm{t}_{\text {minor }}=26.1 \mathrm{~min}$ ). $[\alpha]_{\mathrm{D}}{ }^{20}=+159.3^{\circ}\left(\mathrm{c}=0.108, \mathrm{CH}_{2} \mathrm{Cl}_{2}, 91 \%\right.$ ee; $56 \%$ yield $) .{ }^{1} \mathrm{H}$ NMR $\delta(600 \mathrm{MHz}$, $\left.\mathrm{CDCl}_{3}\right): 8.28$ (d, $\left.J=8.8,2 \mathrm{H} ; \mathrm{Ph}-\mathrm{H}\right), 7.63(\mathrm{~d}, J=8.7,2 \mathrm{H} ; \mathrm{Ph}-\mathrm{H}), 5.55$ (dd, $J=$ 12.1, 4.1, 1H; PhCH-O), 5.25 (d, $J=1.1,1 \mathrm{H} ;=\mathrm{CH}), 4.03$ (m, 2H; $\mathrm{OCH}_{2}$ ), 2.78 (ddd, $J=17.1,12.1$, $\left.1.2,1 \mathrm{H} ; \mathrm{CH}_{\mathrm{A}} \mathrm{H}_{\mathrm{B}}\right), 2.67\left(\mathrm{dd}, J=17.1,4.1,1 \mathrm{H} ; \mathrm{CH}_{\mathrm{A}} \mathrm{H}_{\mathrm{B}}\right), 1.42\left(\mathrm{t}, J=7.0,3 \mathrm{H} ; \mathrm{CH}_{3}\right) .{ }^{13} \mathrm{C}$ NMR $\delta$ $\left(150 \mathrm{MHz}, \mathrm{CDCl}_{3}\right): 171.3,166.3,148.2,145.5,126.9,124.2,91.0,76.0,65.5,35.3,14.2$. HRMS: $(\mathrm{M}+\mathrm{H})^{+}$, calcd 264.0866, found 264.0874 .
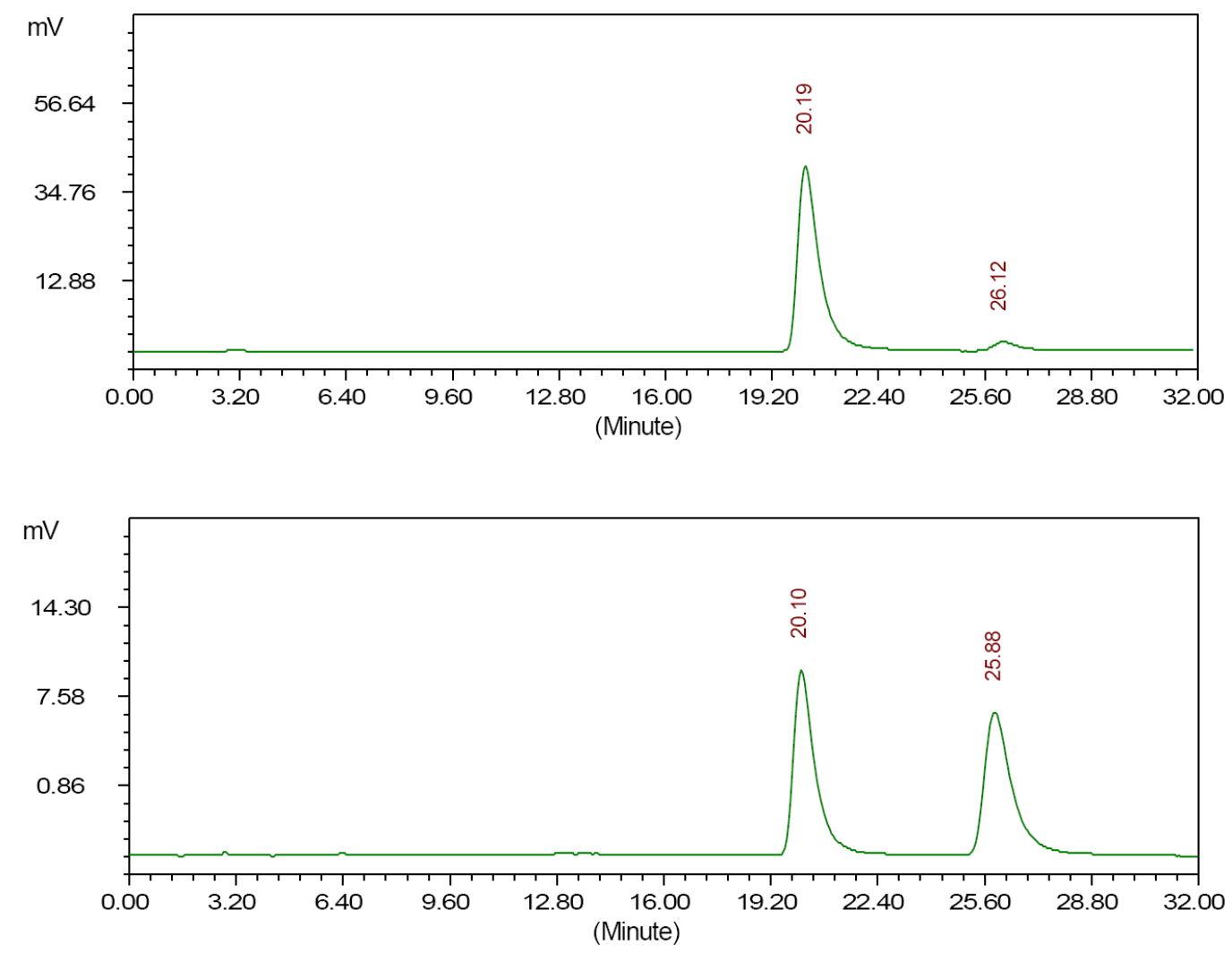


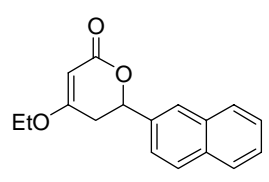

\section{4-ethoxy-5,6-dihydro-6-(naphthalen-3-yl)pyran-2-one (3l)}

The ee was determined by HPLC using a Chiralpak AD-H column $(92 / 8$ hexane/isopropanol; flow rate $1.0 \mathrm{~mL} / \mathrm{min}$; $\mathrm{t}_{\text {major }}=19.5 \mathrm{~min}, \mathrm{t}_{\text {minor }}=24.5 \mathrm{~min}$ ).

$[\alpha]_{\mathrm{D}}{ }^{20}=+145.5^{\circ}\left(\mathrm{c}=0.176, \mathrm{CH}_{2} \mathrm{Cl}_{2}, 96 \%\right.$ ee; $61 \%$ yield $) .{ }^{1} \mathrm{H} \mathrm{NMR} \delta(600 \mathrm{MHz}$, $\mathrm{CDCl}_{3}$ ): 7.91-7.86 (m, 4H; Ar-H), 7.63 (m, 3H; Ar-H), 5.61 (dd, J= 12.1, 3.8, 1H; ArCH-O), 5.26 (s, $1 \mathrm{H} ;=\mathrm{CH}), 4.02\left(\mathrm{~m}, 2 \mathrm{H} ; \mathrm{OCH}_{2}\right), 2.91\left(\mathrm{dd}, J=17.2,12.1,1 \mathrm{H} ; \mathrm{CH}_{\mathrm{A}} \mathrm{H}_{\mathrm{B}}\right), 2.70(\mathrm{dd}, J=17.2,3.8,1 \mathrm{H}$; $\left.\mathrm{CH}_{\mathrm{A}} \mathrm{H}_{\mathrm{B}}\right), 1.42\left(\mathrm{t}, J=7.0,3 \mathrm{H} ; \mathrm{CH}_{3}\right) .{ }^{13} \mathrm{C} \mathrm{NMR} \delta\left(150 \mathrm{MHz}, \mathrm{CDCl}_{3}\right): 171.92,167.27,135.92,133.46$, 133.32, 128.79, 128.35, 127.93, 126.69, 126.66, 125.32, 123.69, 91.04, 65.18, 35.53, 14.23. HRMS: $(\mathrm{M}+\mathrm{H})^{+}$, calcd 269.1172 , found 269.1165 .
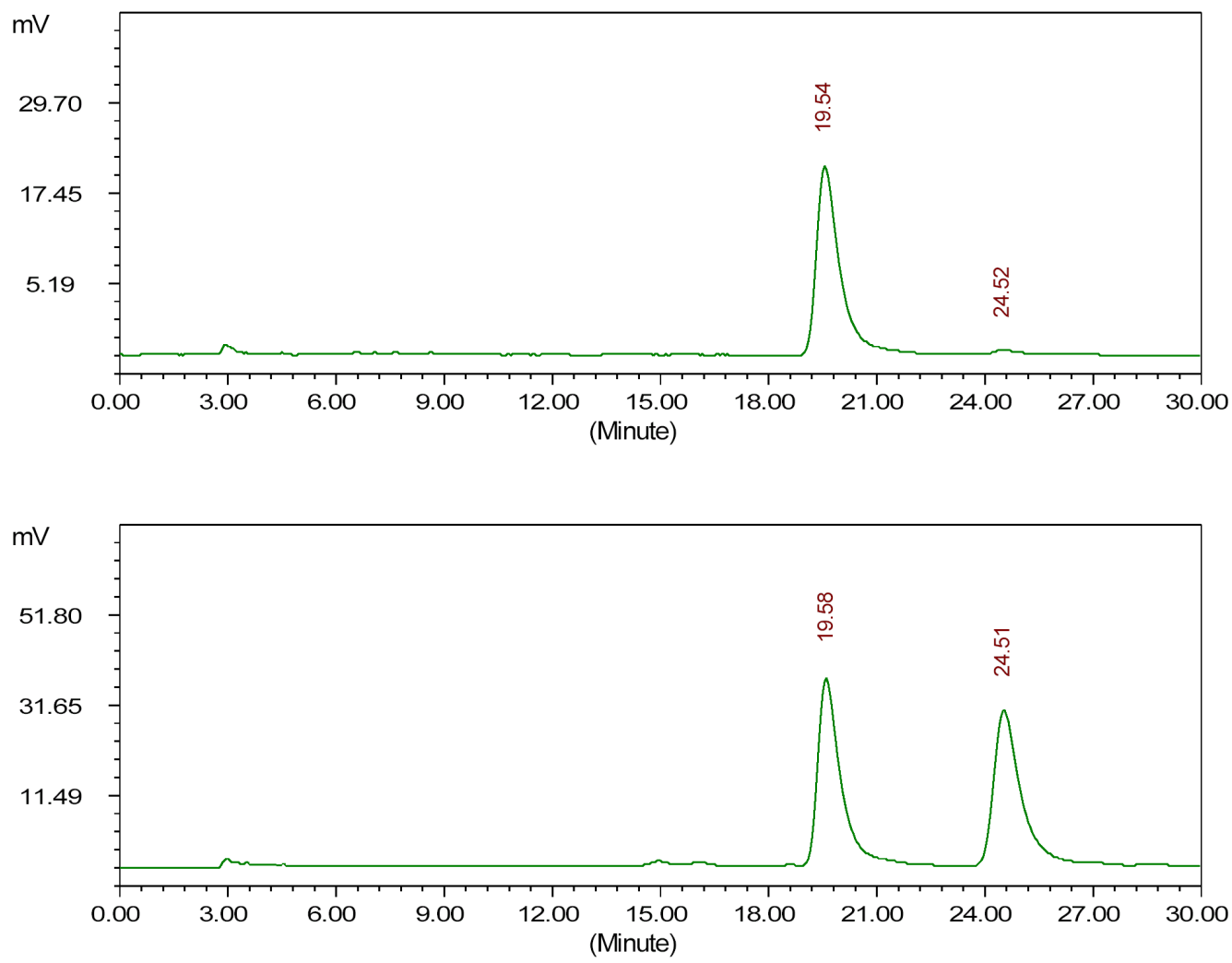

\section{Copies of ${ }^{1} \mathrm{H}$ NMR and ${ }^{13} \mathrm{C}$ NMR spectra of some new compounds}




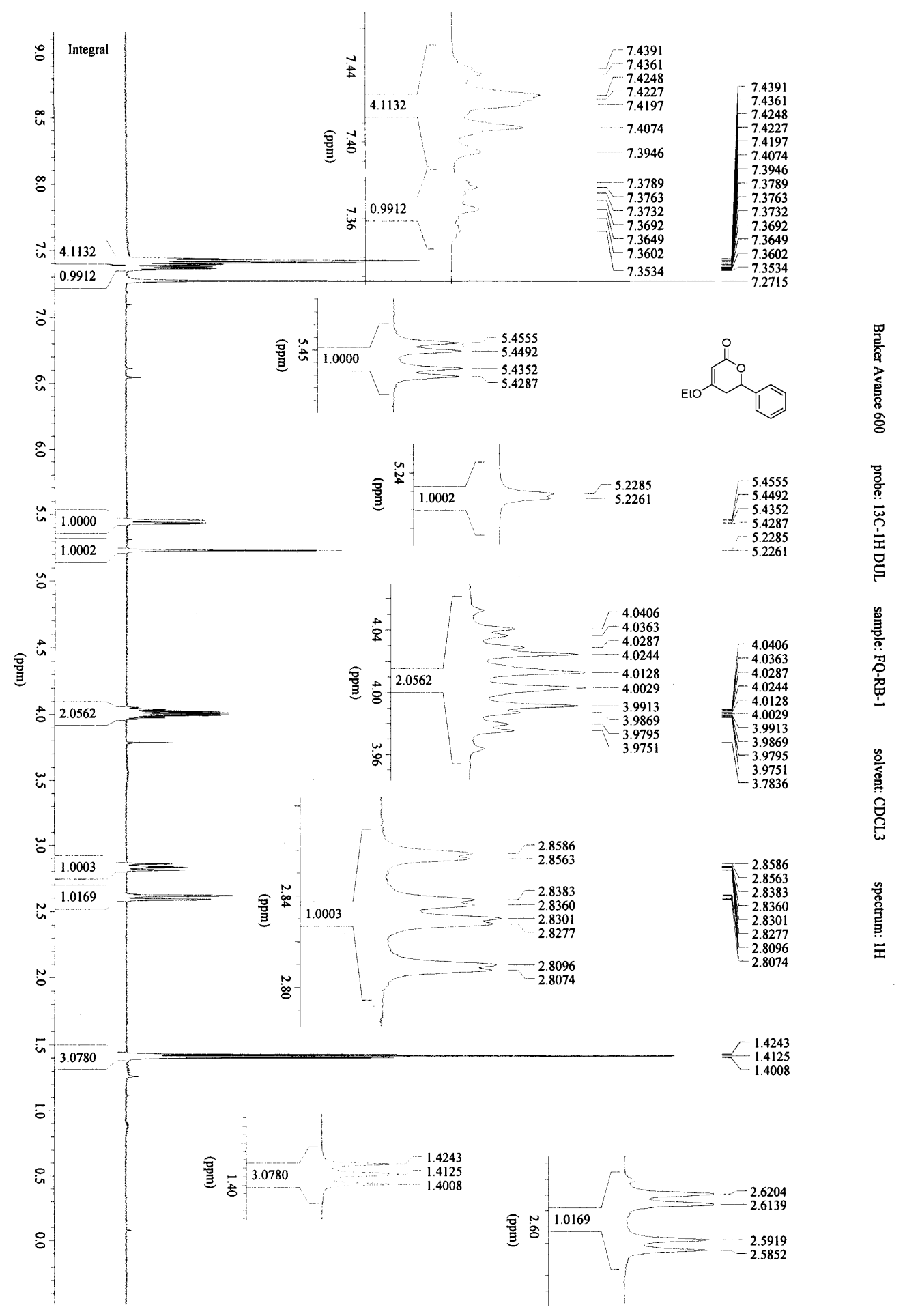




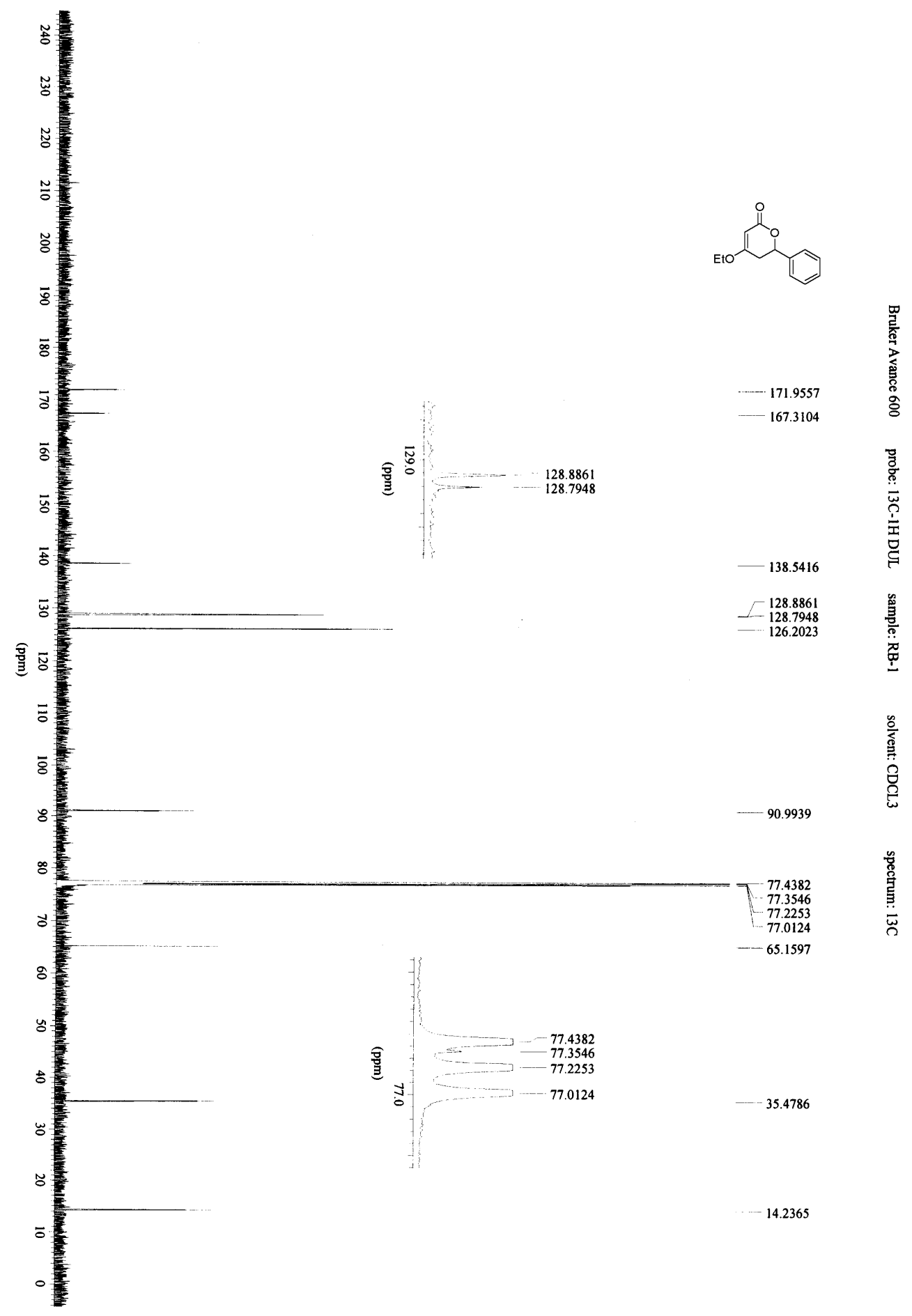




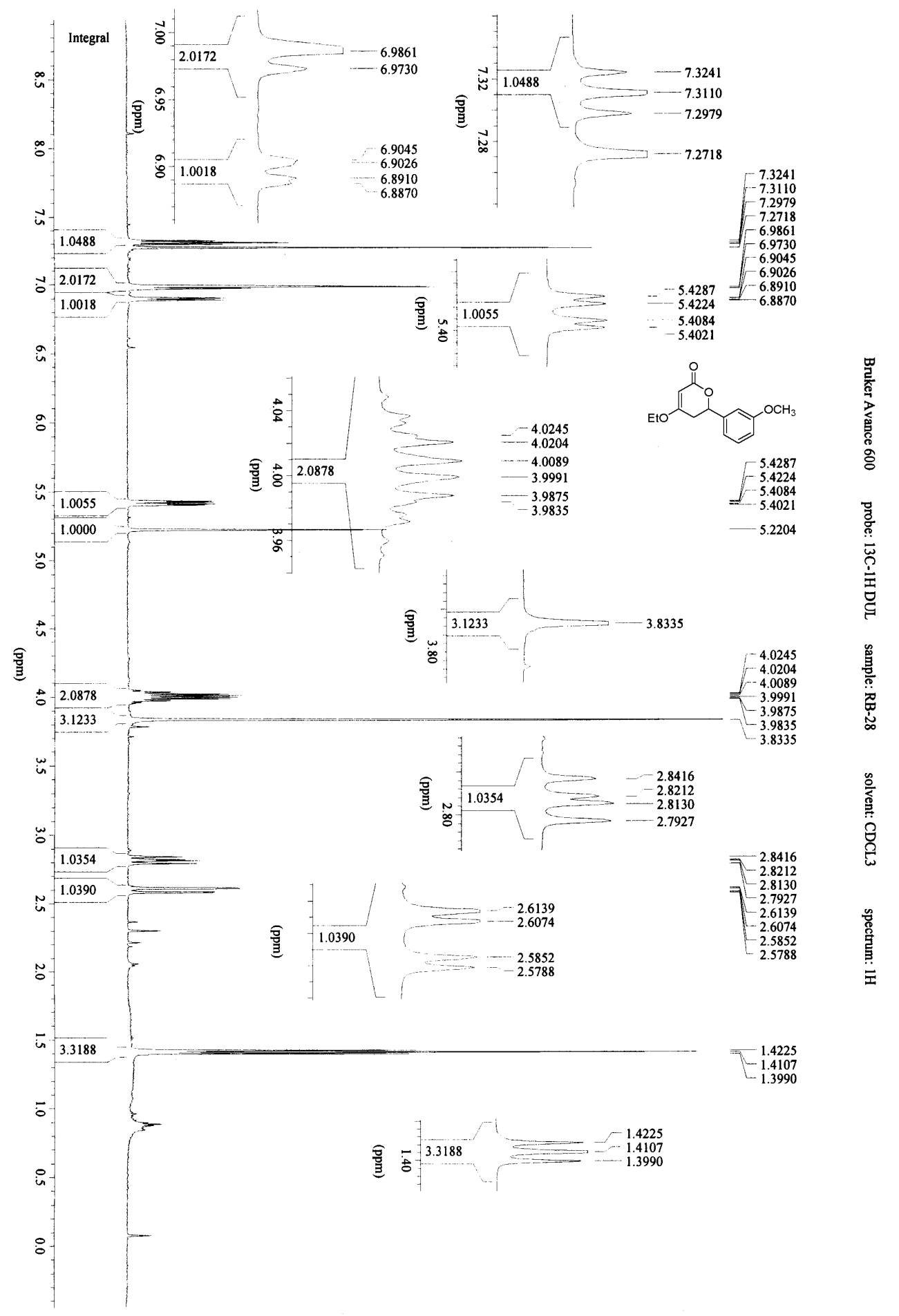




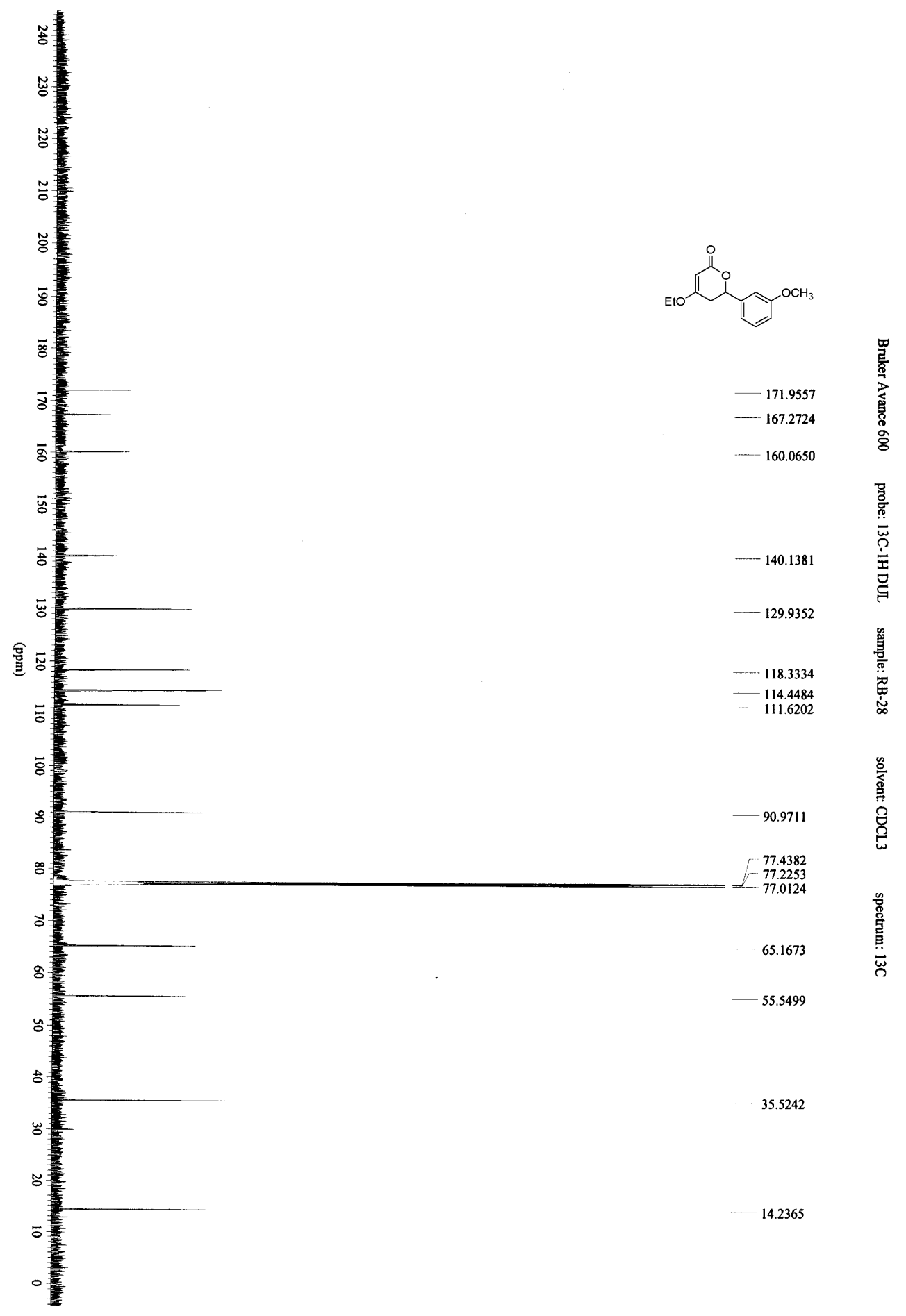




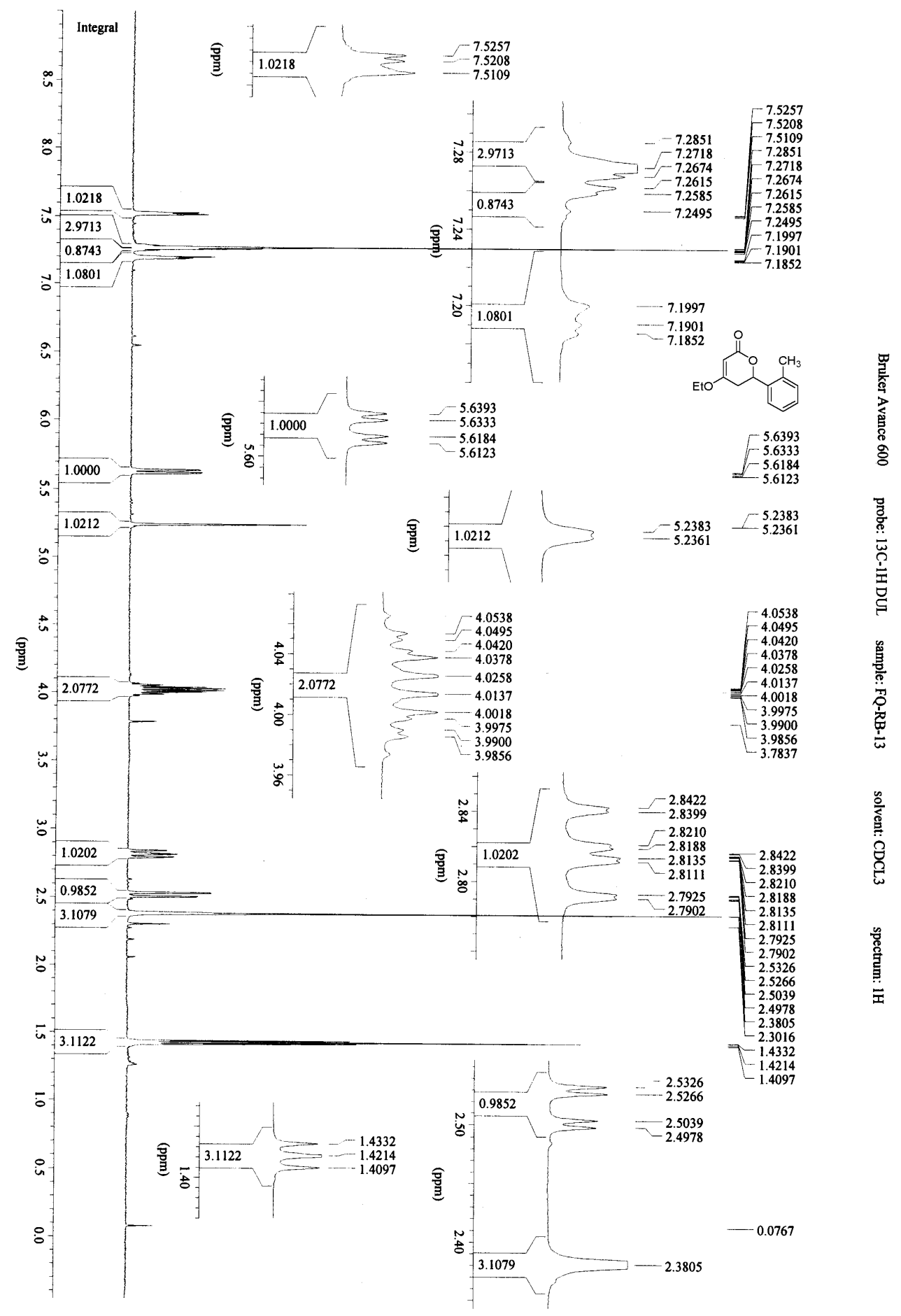




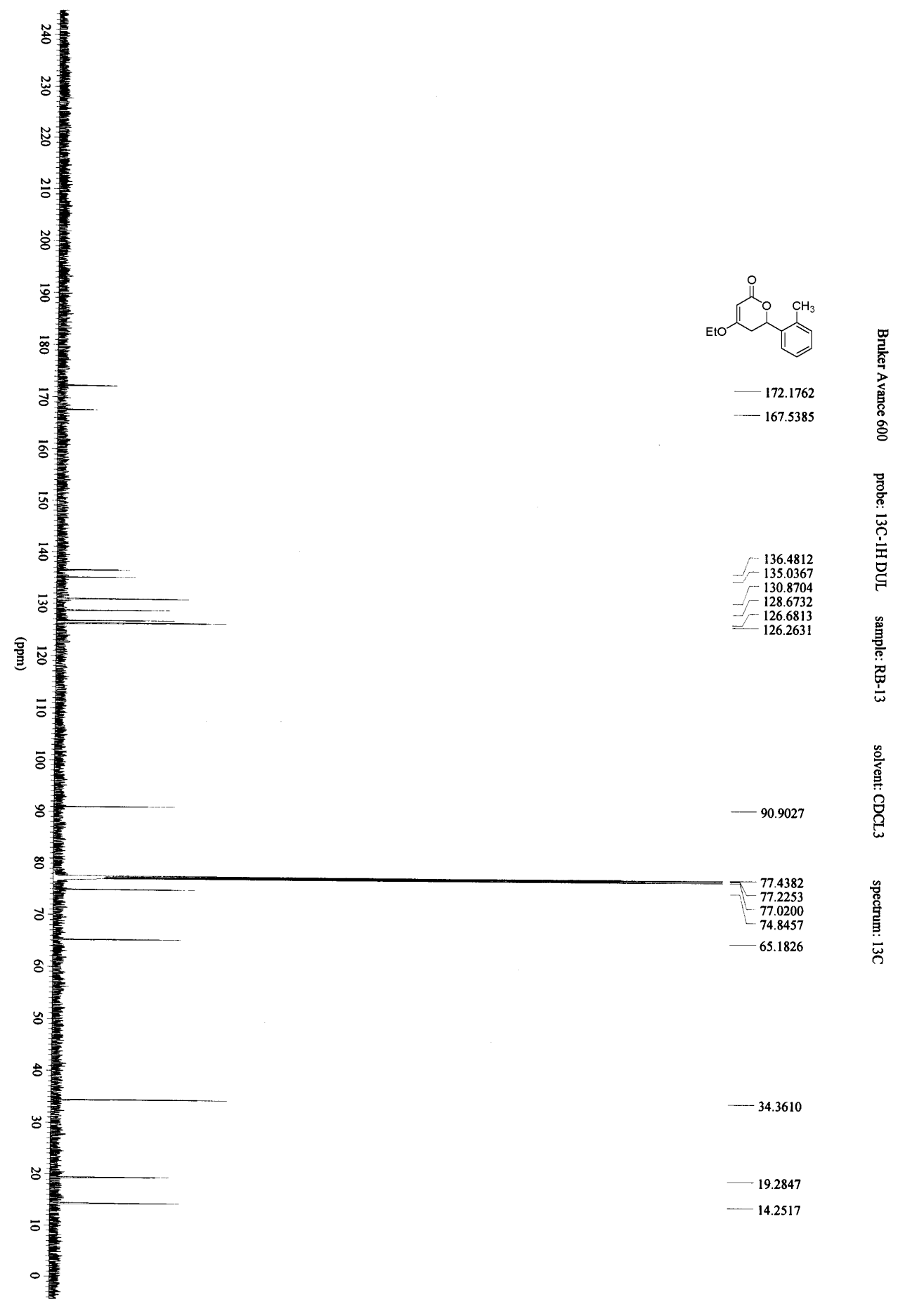




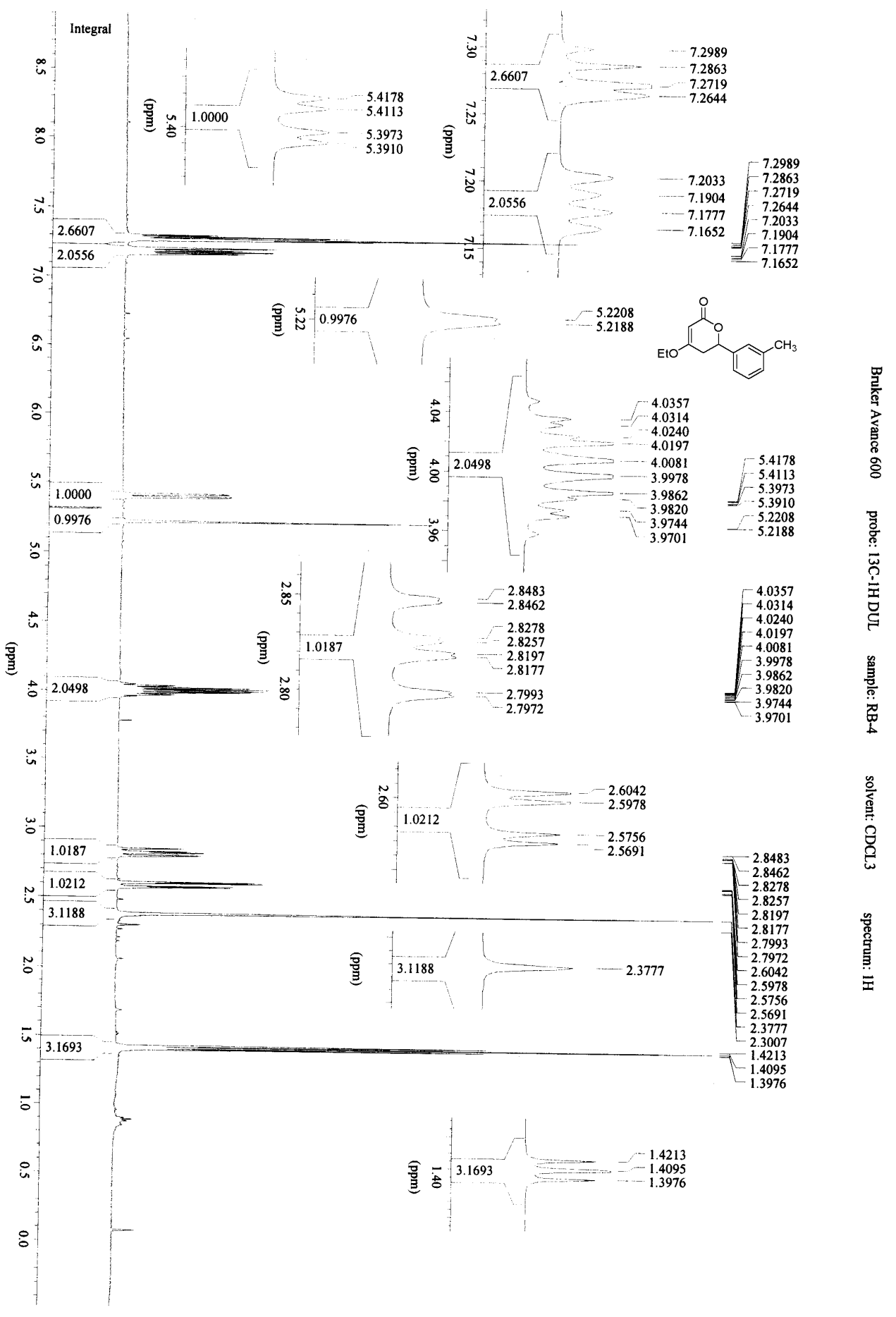




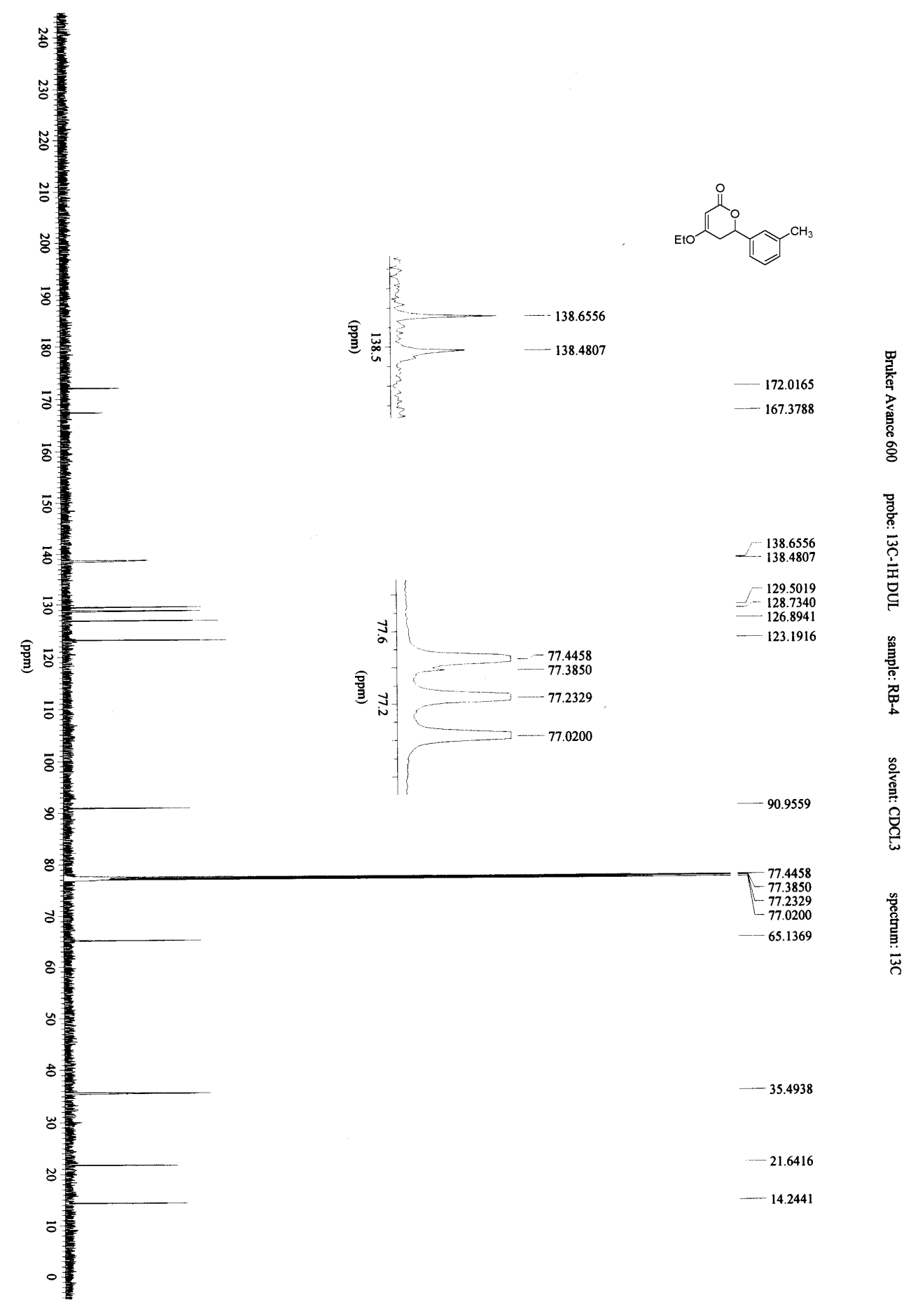




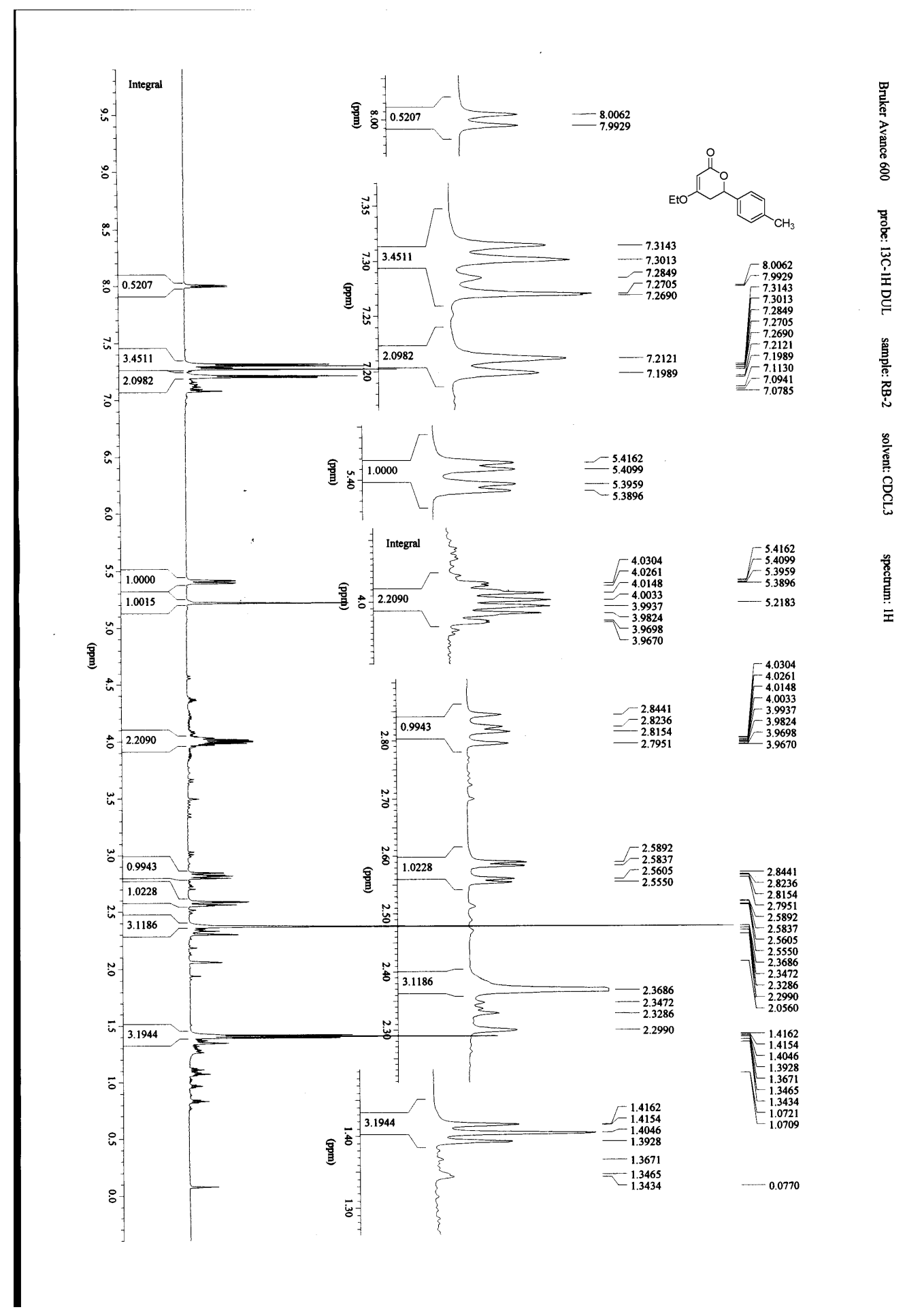




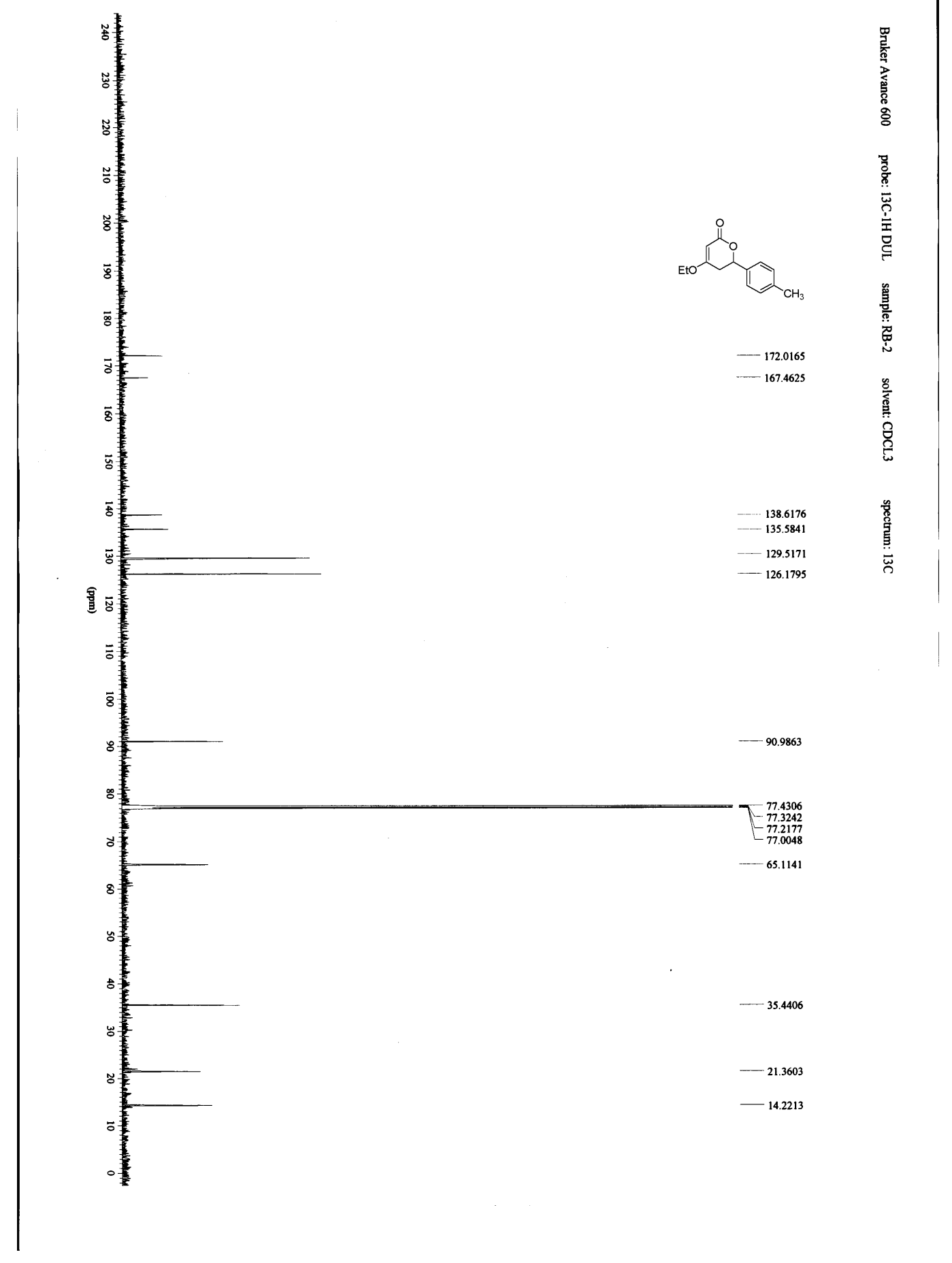




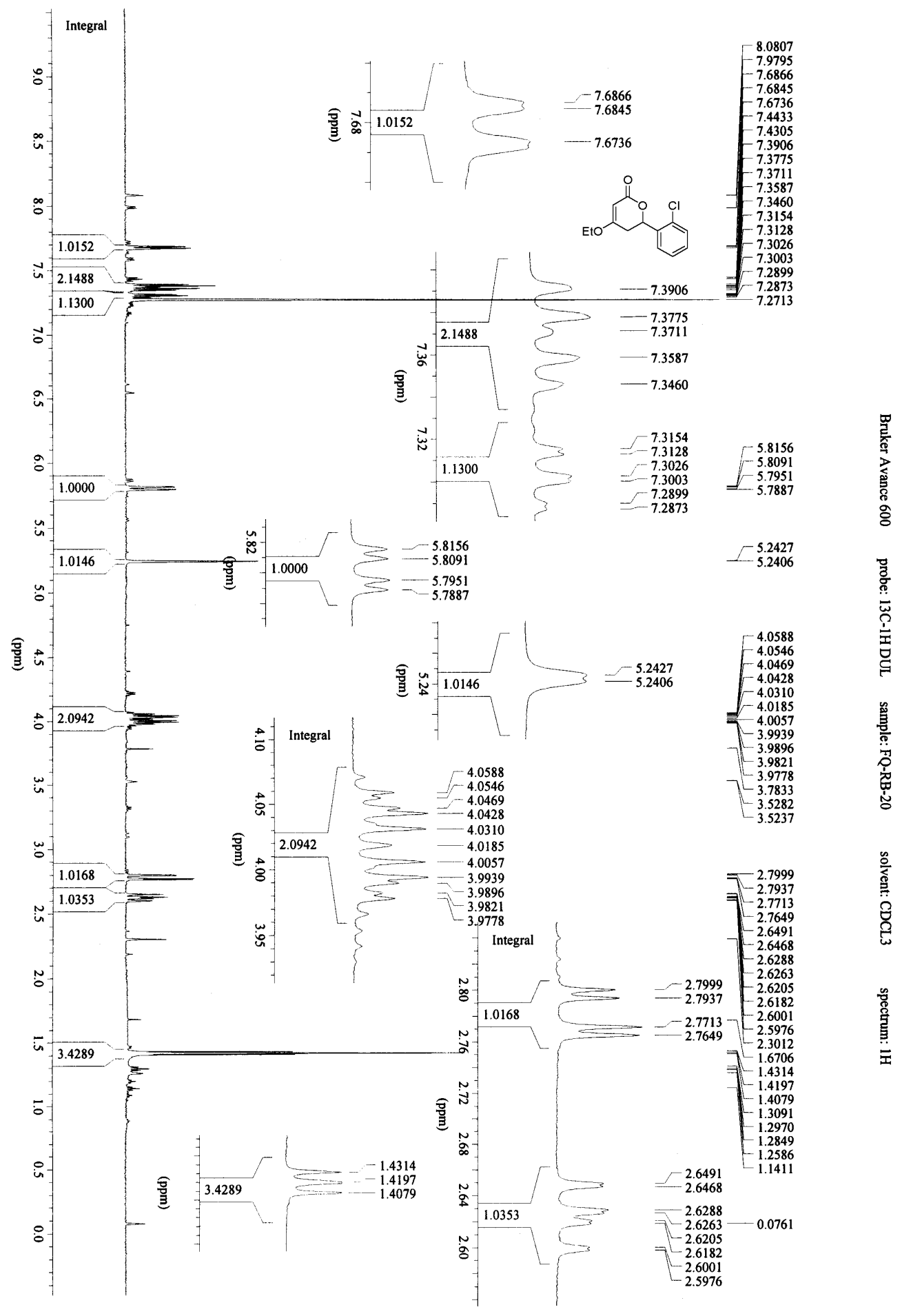




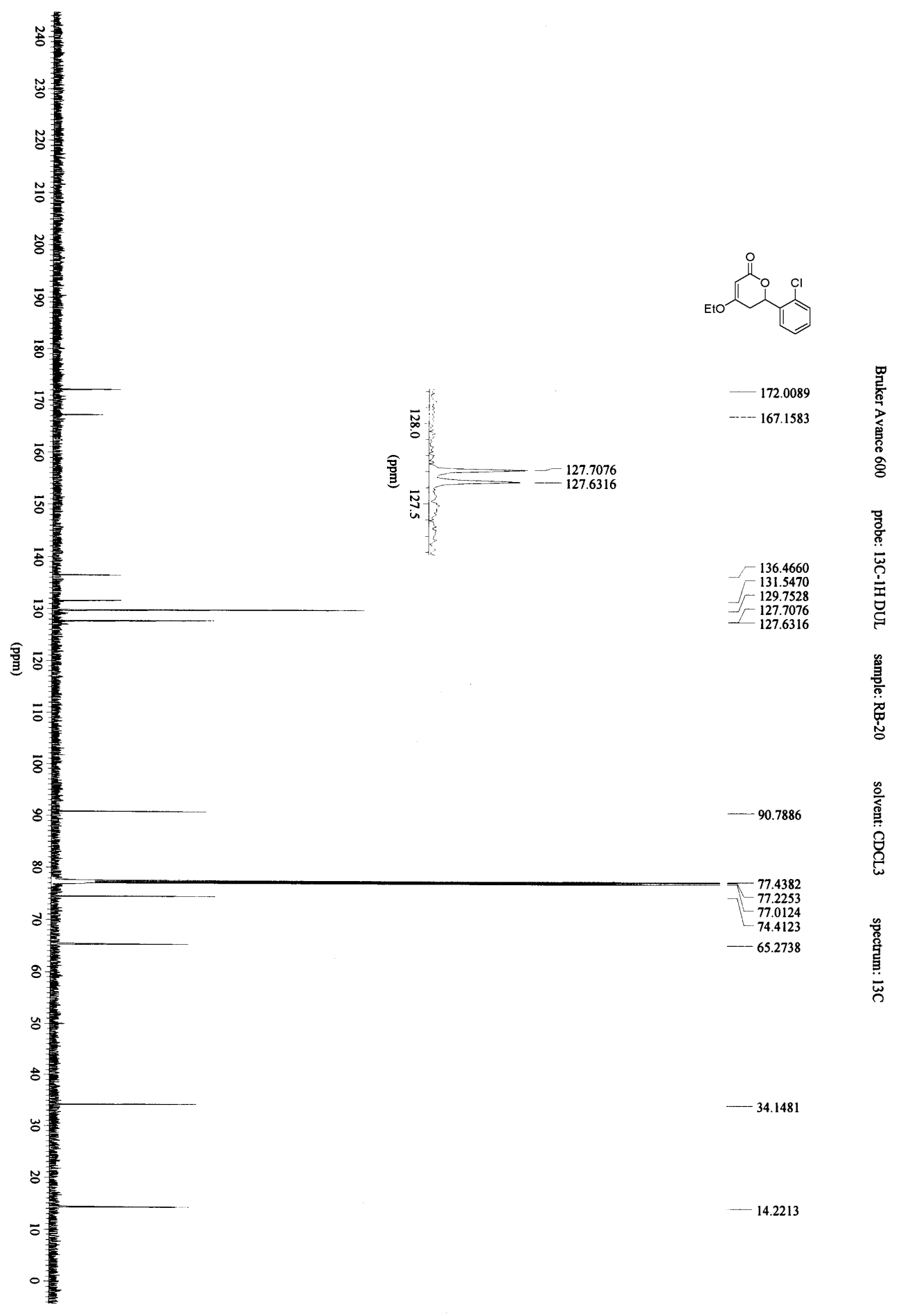




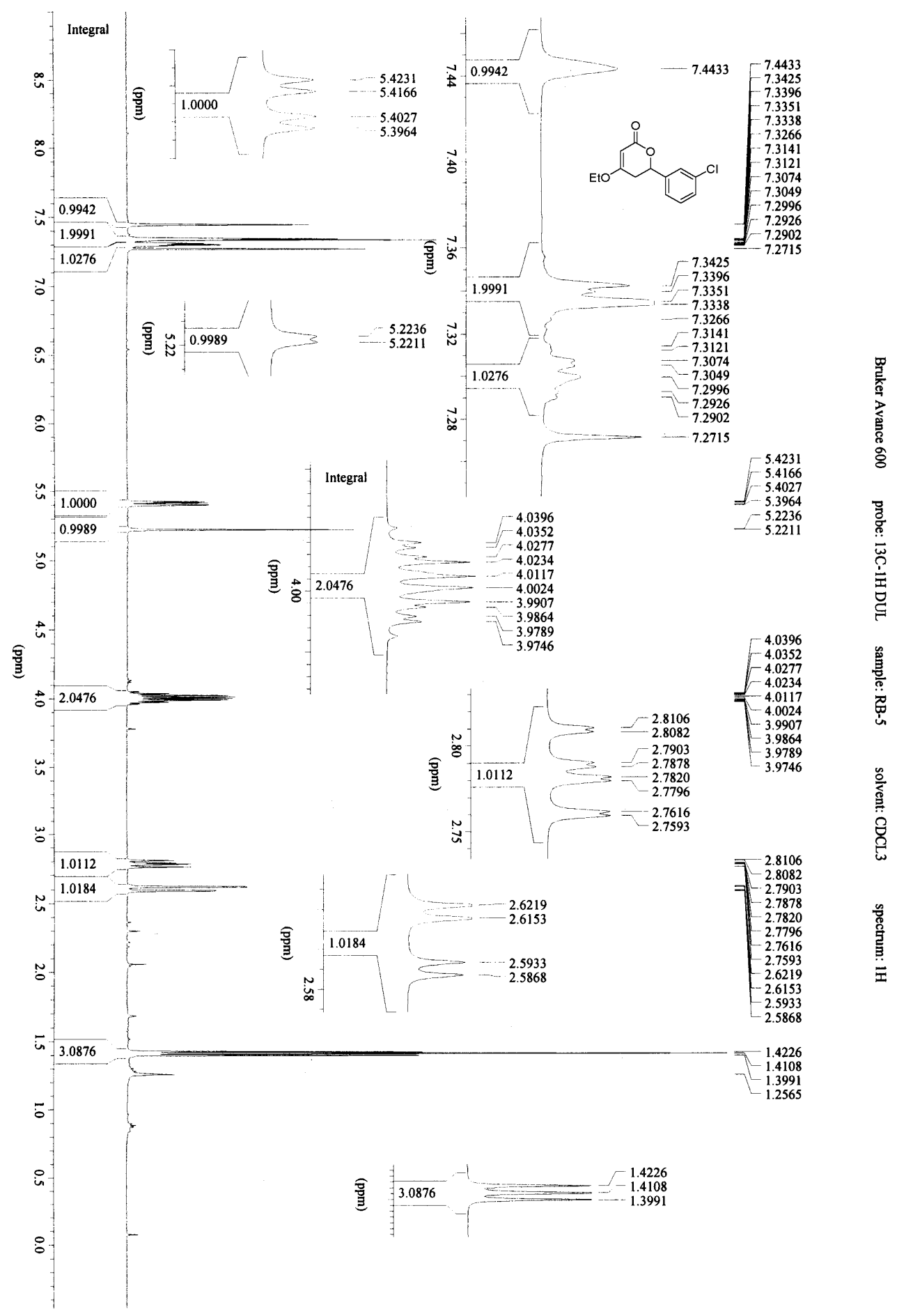




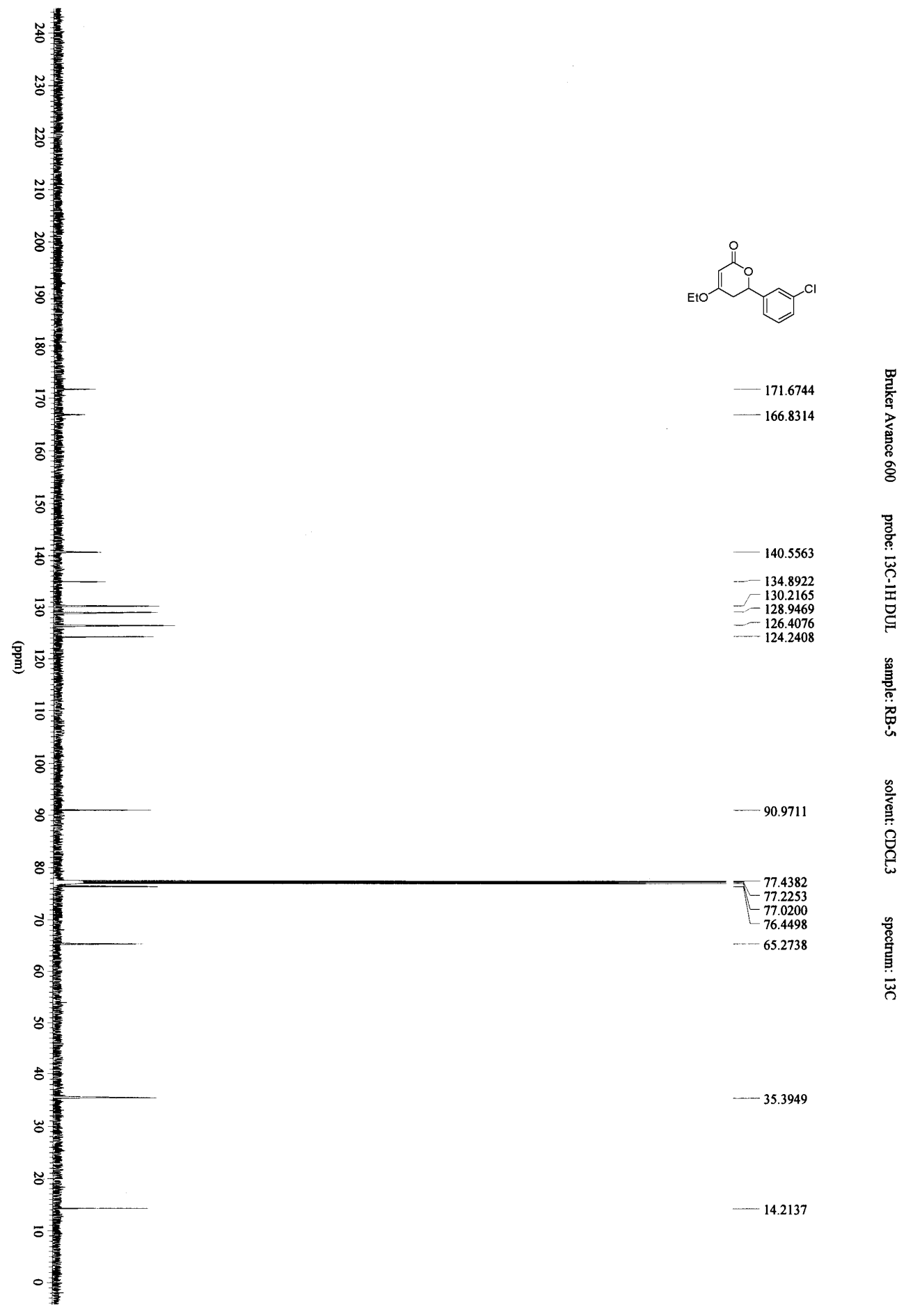




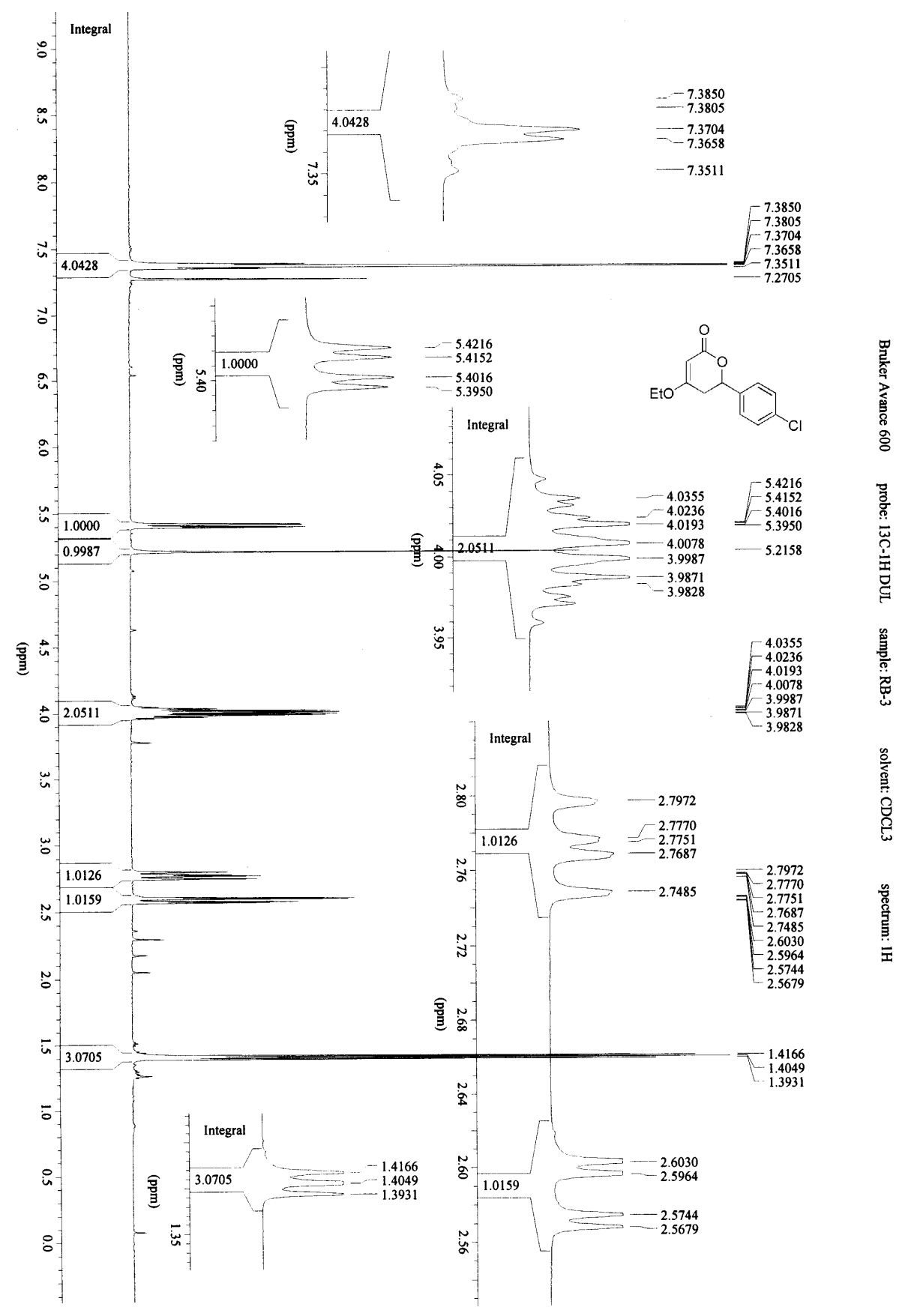




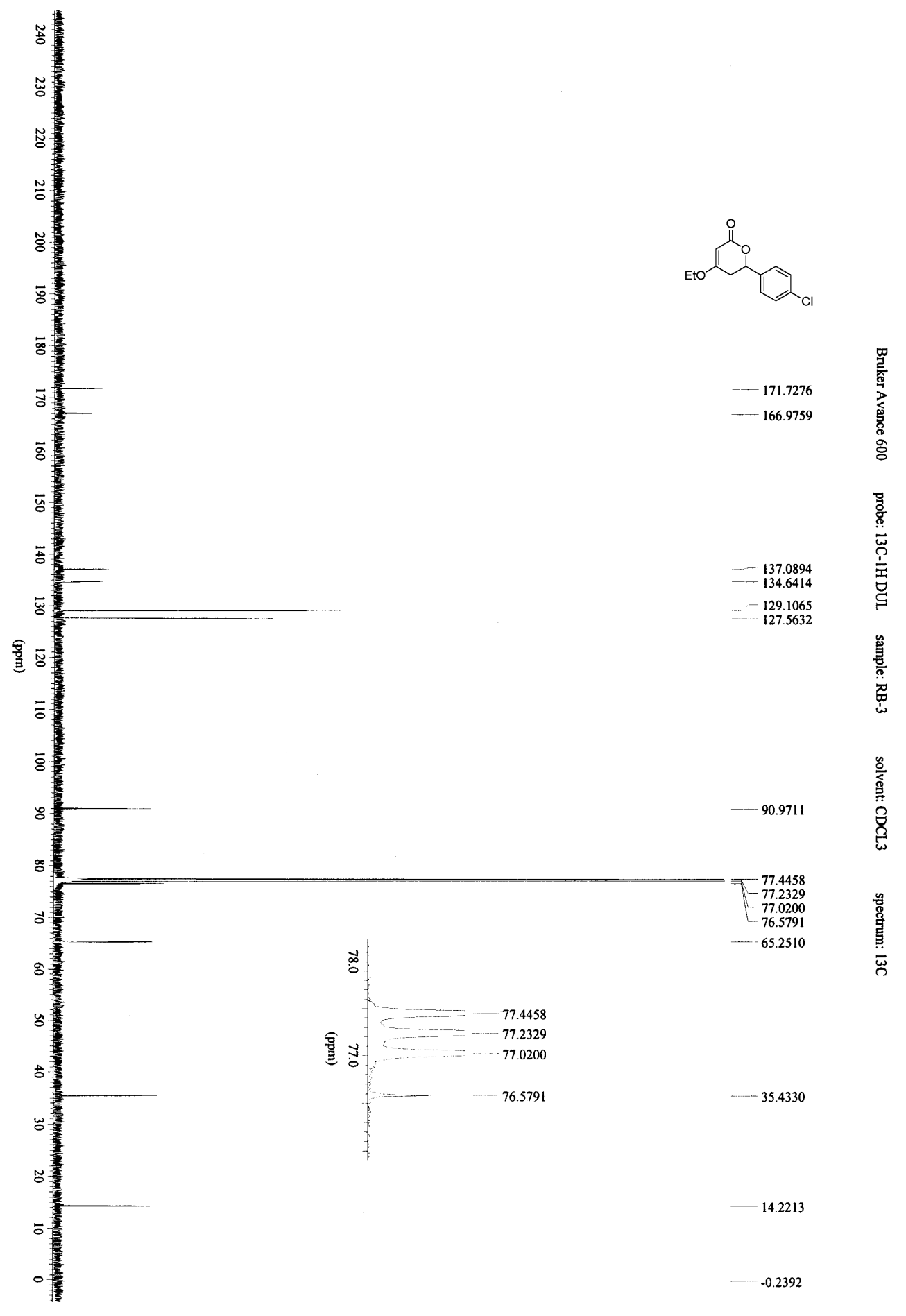




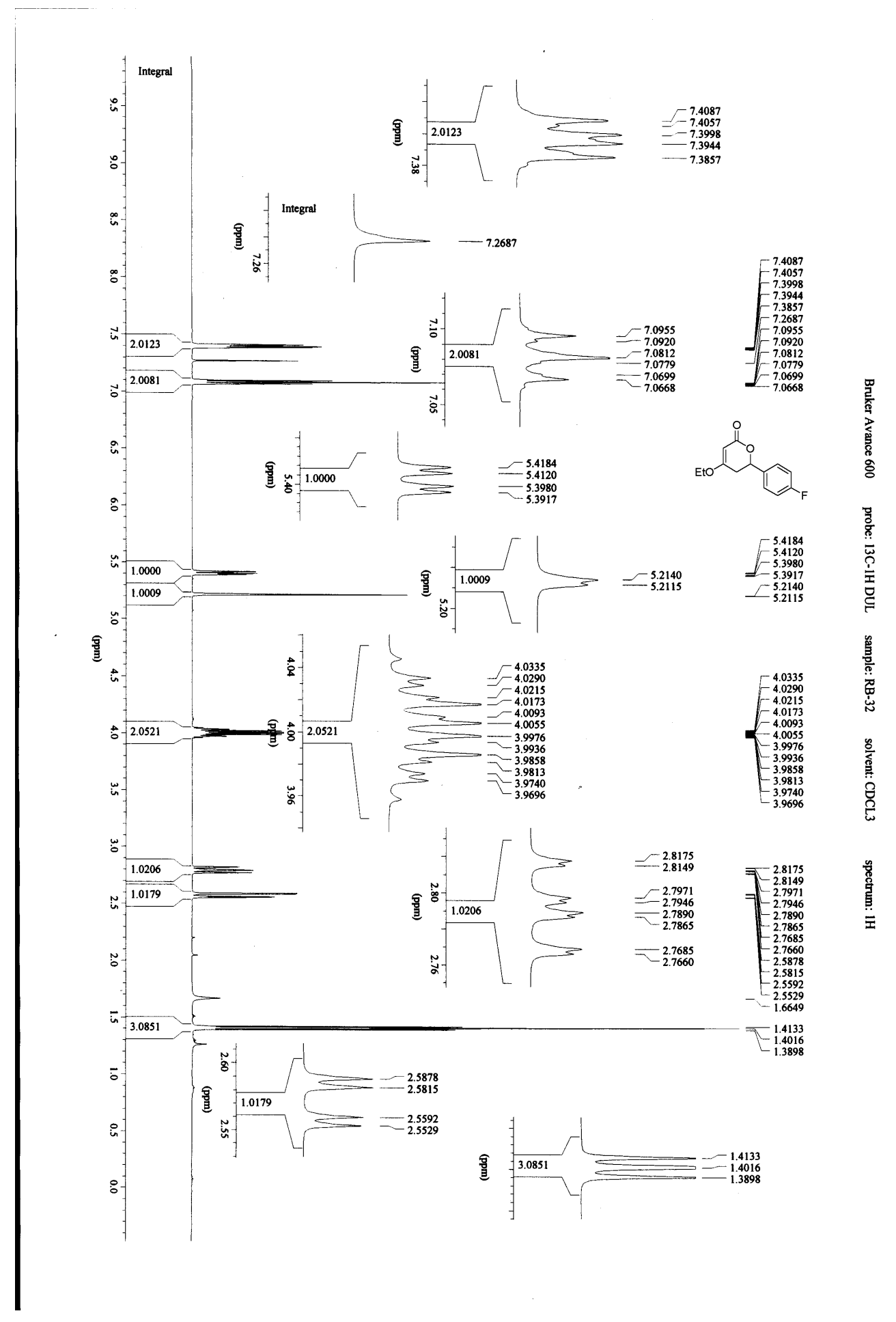




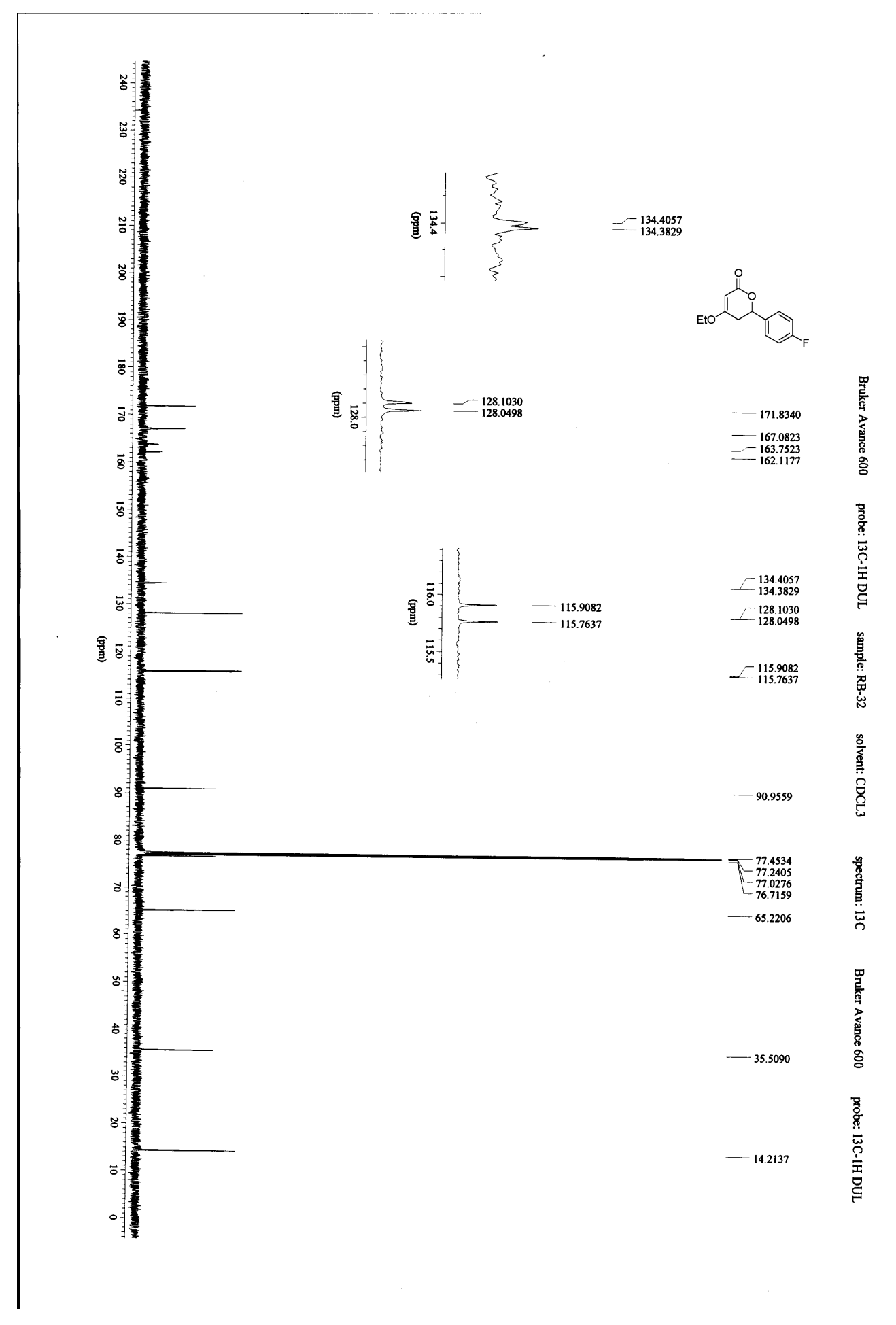




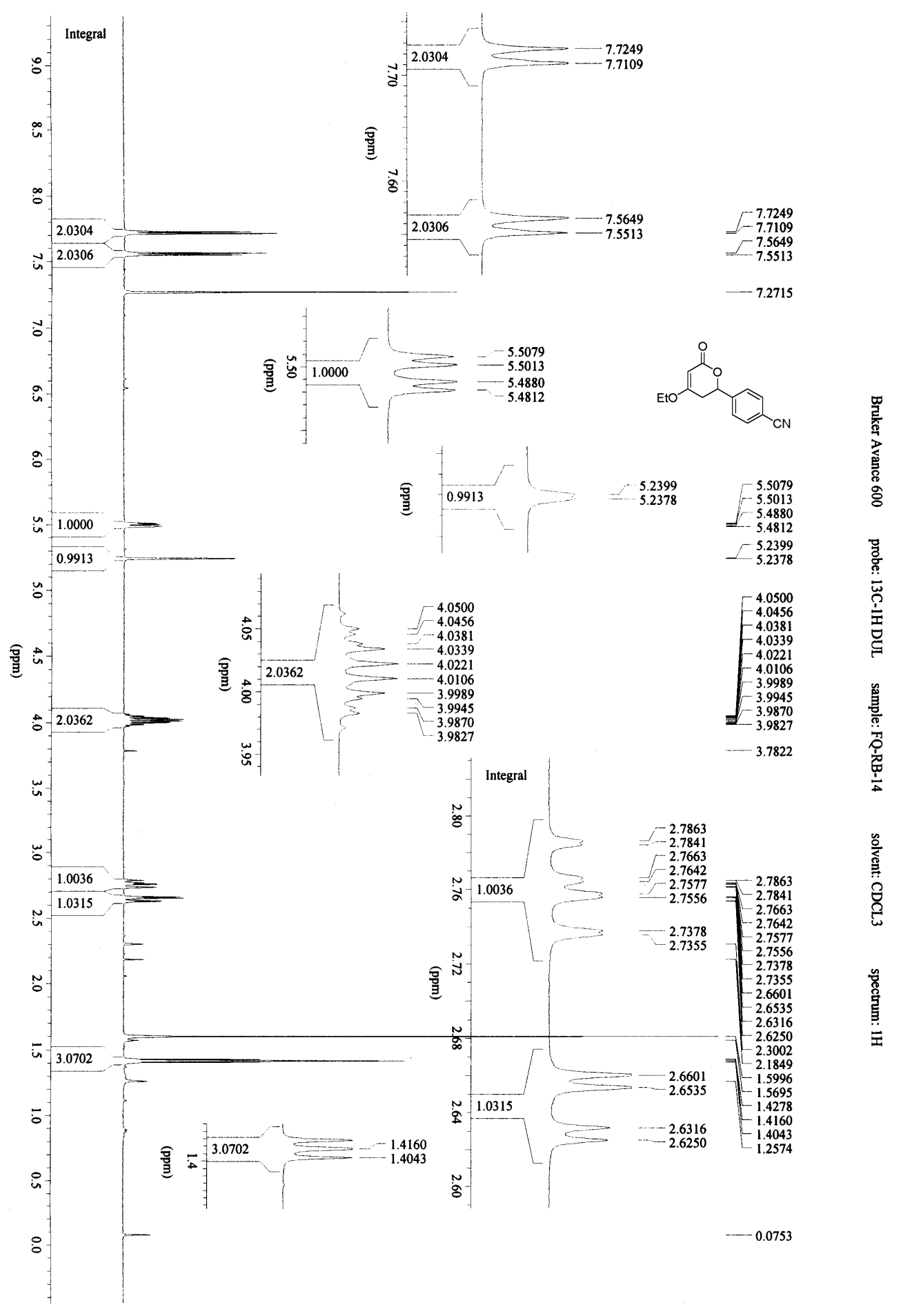




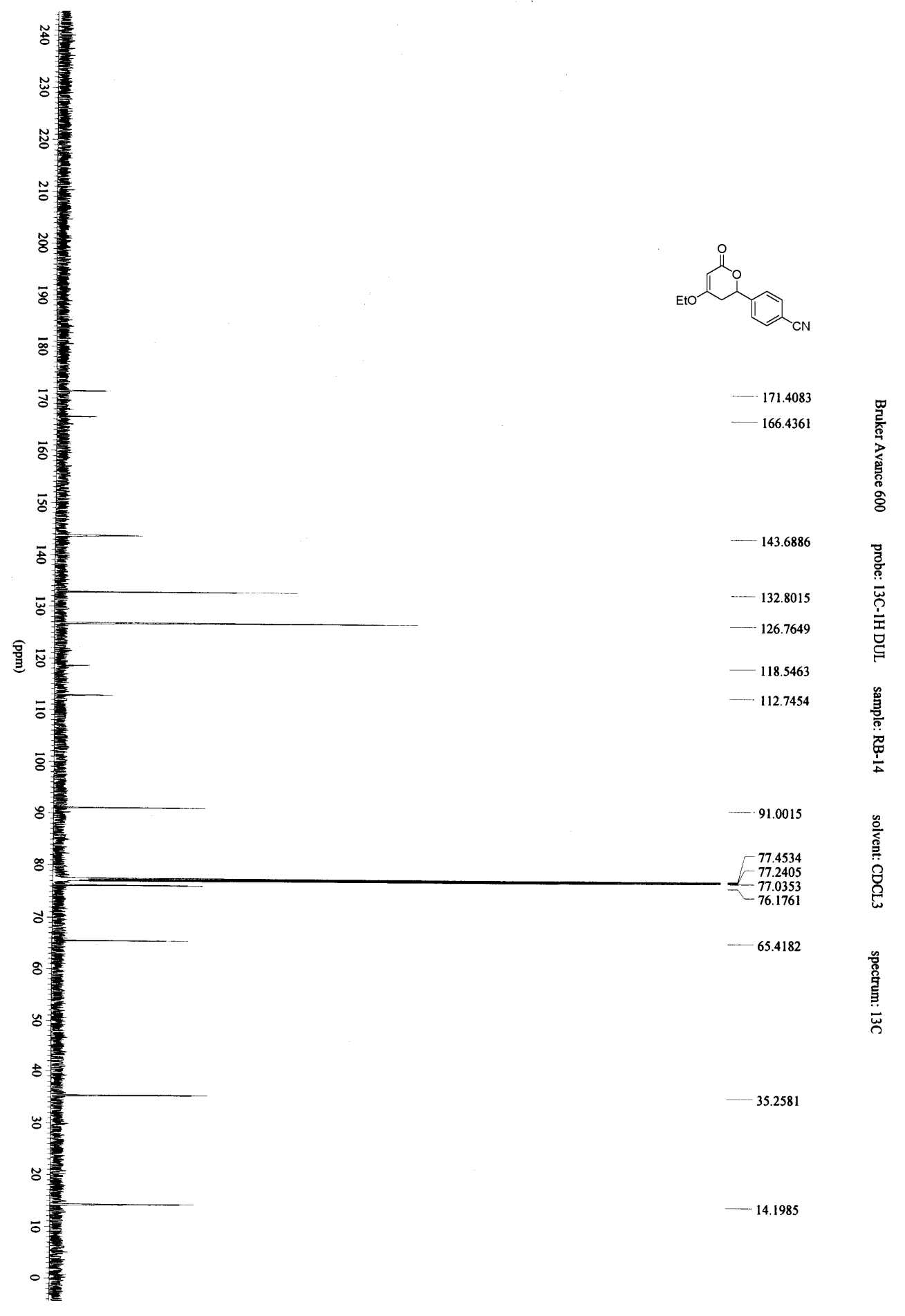




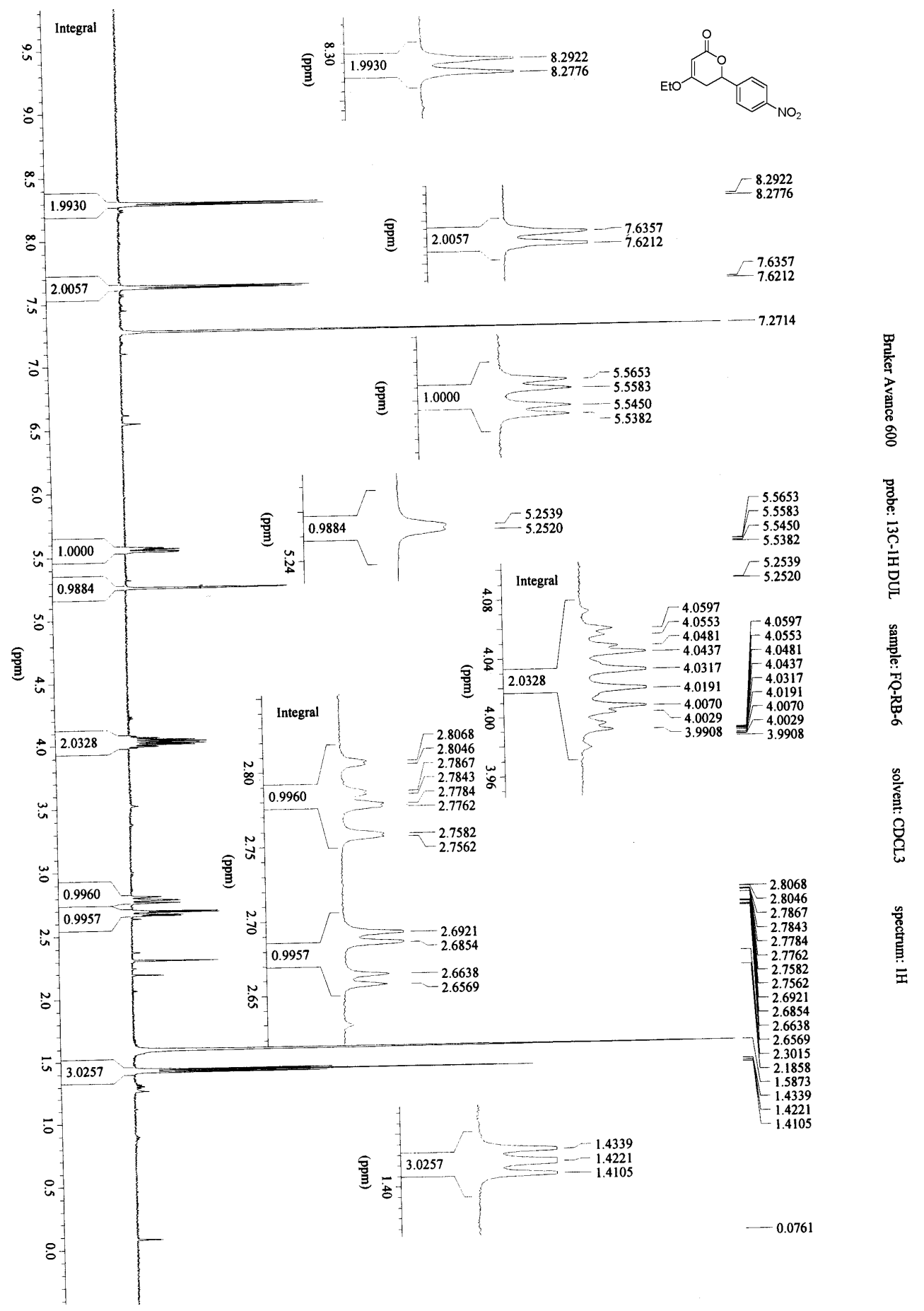




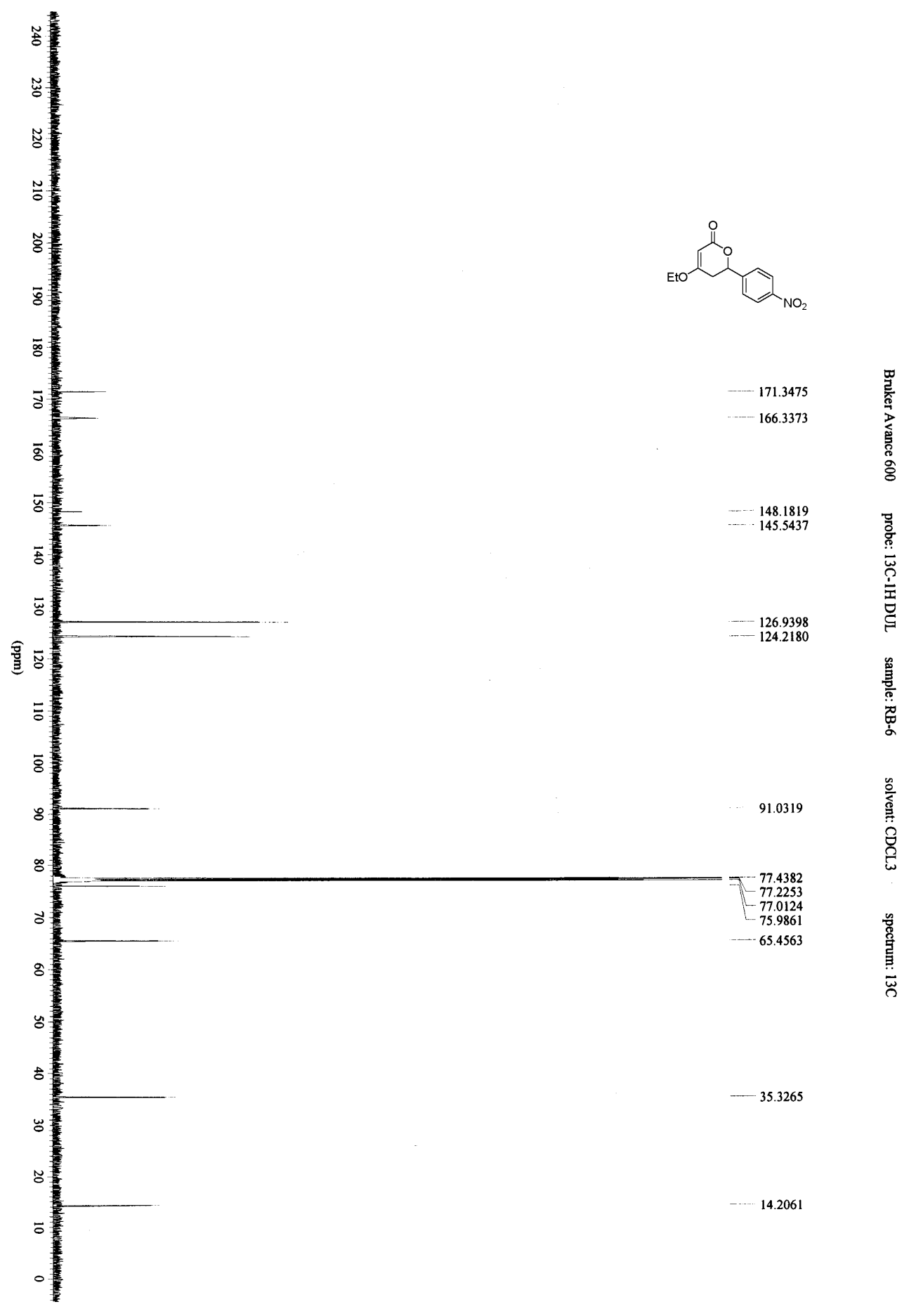




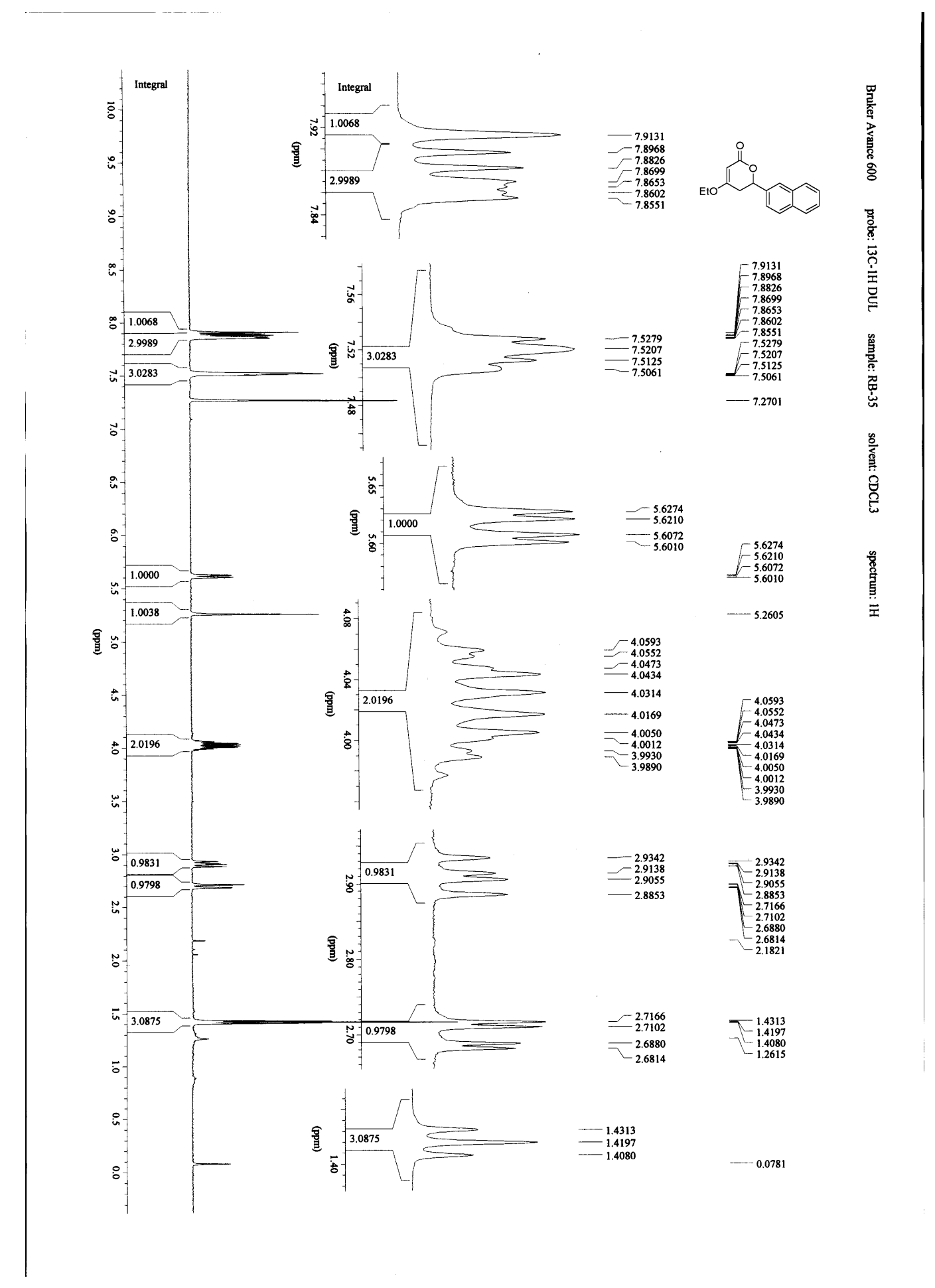




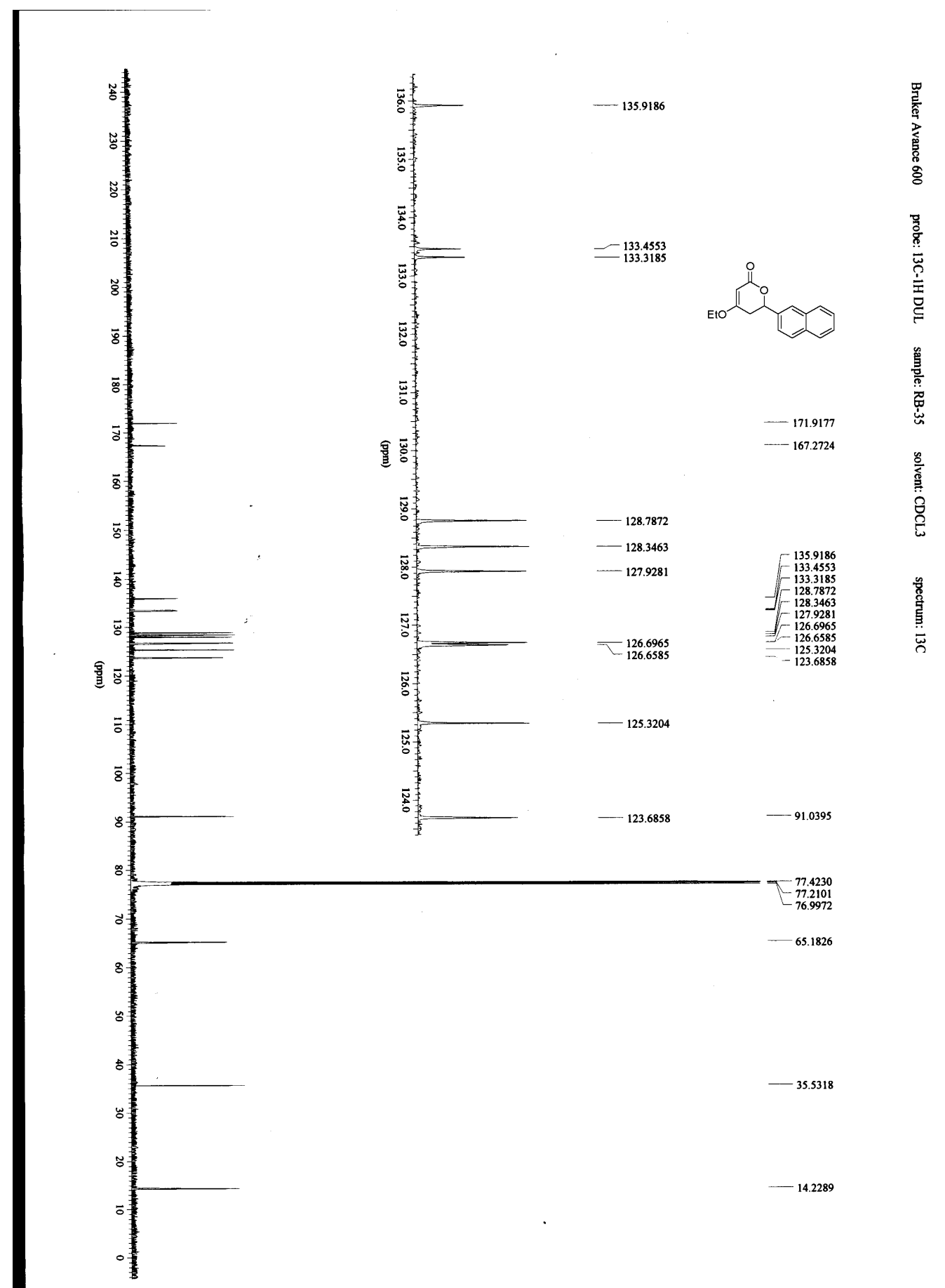

